TRANSACTIONS OF THE

AMERICAN MATHEMATICAL SOCIETY

Volume 182, August 1973

\title{
INVERSE SEMIGROUPS WHICH ARE SEPARATED OVER A SUBSEMIGROUP
}

\author{
BY
}

\author{
D. B. McALISTER( (1)
}

ABSTRACT. An inverse semigroup $T$ is separated over a subsemigroup $S$ if $T$ is generated, as an inverse semigroup, by $S$ and for each $a, b \in S$ there exists $x \in S a \cap S b$ such that $a^{-1} a b^{-1} b=x^{-1} x$ and dually for right ideals. For example, if $T$ is generated as an inverse semigroup by a semigroup $S$ whose principal left and right ideals form chains under inclusion, then $T$ is separated over $S$. In this paper we investigate the structure of inverse semigroups $T$ which are separated over subsemigroups $S$.

The structure theory of inverse semigroups has been the object of much study over recent years with particular attention being paid to 0-bisimple and 0-simple inverse semigroups ([2], [9], [10], [11], [13], for example). These papers attempted to determine the structure of various 0 -bisimple or 0 -simple inverse semigroups directly in terms of groups and semilattices. However the degree of complication involved even in these cases leads one to suspect that this is, in general, a futile task although it is possible in some cases.

In a general sense, the structure of inverse semigroups is determined by its semilattice of idempotents and a semilattice of groups. This is a consequence of a theorem of Munn [11] which shows that the maximal fundamental homomorphic image $S / \mu$ of an inverse semigroup $S$ is a full subsemigroup of the semigroup $T_{E}$ of isomorphisms between the principal ideals of the semilattice $E$ of idempotents of $S$. The canonical homomorphism $\mu: S \rightarrow S / \mu$ is idempotent separating so its kernel is a semilattice of groups. The problem of constructing idempotent separating extensions of semilattices of groups by inverse semigroups has been solved, theoretically at least, by D'Alarcao [4] and Coudron [3] so that one could, in principle, construct all inverse semigroups if one could construct all fundamental inverse semigroups; the latter, however, remain a mystery.

Received by the editors April 10, 1972.

AMS (MOS) subject classifications (1970). Primary $20 \mathrm{M} 10$.

Key words and phrases. Inverse semigroup, shift representation, free inverse semigroup, naturally quasisemilatticed semigroup, fundamental inverse semigroup, inverse semigroup of (strong) quotients.

(1) This research was supported by NSF Grant GP 27917. 
In this paper, we shall adopt a more internal approach to describing inverse semigroups. Suppose that $\theta$ is a homomorphism of a semigroup $S$ into an inverse semigroup $T$. Then we shall say that $T$ is separated over $S$, by $\theta$, if $T$ is generated as an inverse semigroup by $S \theta$ and, for each $a, b \in S$,

$$
\begin{gathered}
a \theta(a \theta)^{-1} b \theta(b \theta)^{-1}=x \theta(x \theta)^{-1} \text { for some } x \in a S \cap b S, \\
(a \theta)^{-1} a \theta(b \theta)^{-1} b \theta=(y \theta)^{-1} y \theta \text { for some } y \in S a \cap S b .
\end{gathered}
$$

The main aim of this paper is to investigate the structure of an inverse semigroup $T$, which is separated over a semigroup $S$, in terms of $S$. Special cases of this concept have been considered before. For example, let $T$ be a bisimple monoid and let $S$ be the right unit subsemigroup of $T$; if $S$ is right reflexive then $T$ is separated over $S$. Clifford [1] has described the structure of $T$ in terms of $S$. On the other hand, Eberhart and Selden [ 5 ] have described the structure of all one parameter inverse semigroups. Any such semigroup $T$ is separated over a subsemigroup $S$ of the multiplicative semigroup of the positive reals.

Theorem 3.5 gives an explicit method of construction for all fundamental inverse semigroups which are separated over an arbitrary semigroup $S$. Thus, by using D'Alarcao's extension theorem [4] one could, in principle, construct all inverse semigroups which are separated over $S$. We have not been able to do this explicitly without imposing conditions on $S$. A semigroup $S$ is naturally quasisemilatticed if the sets of principal left and right ideals of $S$ form semilattices under inclusion; thus an inverse semigroup is naturally quasisemilatticed. If $S$ is naturally semilatticed and $T$ is separated over $S$ by $\theta$ then, for $a, b \in S$,

$$
\begin{aligned}
& a \theta(a \theta)^{-1} b \theta(b \theta)^{-1}=\left(a \wedge_{r} b\right) \theta\left[\left(a \wedge_{r} b\right) \theta\right]^{-1}, \\
& (a \theta)^{-1} a \theta(b \theta)^{-1} b \theta=\left[\left(a \wedge_{l} b\right) \theta\right]^{-1}\left(a \wedge_{l} b\right) \theta,
\end{aligned}
$$

where, for example, $a \wedge, b$ in $S$ is such that $a S^{1} \cap b S^{1}=(a \wedge, b) S^{1}$. There is thus a universal inverse semigroup $E(S)$ in the category of inverse semigroups which are separated over $S$. An explicit construction and several coordinatisations for $E(S)$ are given in $\$ 4$ while the congruences and ideal structure form the subject matter of $\$ 5$.

Whenever the sets of principal left and right ideals of a semigroup $S$ are chains under inclusion, every inverse semigroup generated, as an inverse semigroup, by a homomorphic image of $S$ is separated over $S$. Hence $E(S)$ is the free inverse semigroup on $S$ and so $S$ can be embedded in an inverse semigroup if and only if it can be embedded in $E(S)$. The last result remains true if $S$ is naturally quasisemilatticed (Theorem 4.6) so that we can use $E(S)$ to obtain a set of necessary and sufficient conditions for the embeddability of such semigroups in inverse semigroups. 
The main tools used in this paper are what we term shift representations of $S$ by one-to-one partial transformations. These representations generalise both the Vagner-Preston representations of inverse semigroups and the regular representations of cancellative semigroups. They are described in $\$ 2$.

The theory undergoes considerable simplification when the semigroup $S$ under consideration is cancellative. It is applied in $\$ 6$ to give necessary and sufficient conditions on a cancellative semigroup so that each element of $I(S)$ should be of the form $a b^{-1} c$ with $a, b, c \in S$; the precise conditions are that the sets of principal left and right ideals of $S$ should be chains under inclusion. The theory is also applied to give a characterisation of the positive cone of a right ordered group.

The final section consists of several examples of inverse semigroups which arise from the general theory. In particular the theory gives a method for constructing 0 -simple inverse semigroups in which $\mathfrak{D} \neq \mathscr{G}$. The $\mathscr{D}$-classes in these semigroups are traversed by a semigroup but no $\mathscr{D}$-class is a subsemigroup so that the 0-simple inverse semigroups obtained here are, in a sense, dual to those considered by Munn [12].

1. Embedding a semigroup in an inverse semigroup. If $S$ is any semigroup, it follows from general categorical considerations, or from [8], that there is an inverse semigroup $I(S)$ and a homomorphism $\eta: S \rightarrow I(S)$ with the following property: given any homomorphism $\theta$ of $S$ into an inverse semigroup $T$, there is a unique homomorphism $\psi: I(S) \rightarrow T$ such that the diagram

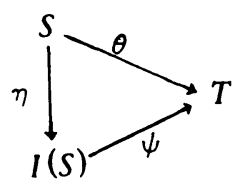

commutes. The semigroup $I(S)$ is called the free inverse semigroup on $S$. One of the aims of this paper is to investigate the structure of $I(S)$ and some related semigroups when the ideal structure of $S$ has certain special properties; in particular, when the sets of principal left and right ideals of $S$ form chains under inclusion.

It follows easily from the functorial properties of $S^{1}, S^{0}$ and $I(S)$ that $I\left(S^{1}\right)$ and $I(S)^{1}$ and $I\left(S^{0}\right)$ and $I(S)^{0}$ are naturally isomorphic. Hence, in studying the relationships between $S$ and $I(S)$ we may, without loss of generality, assume that $S$ has a zero and an identity. We shall assume the latter throughout this paper.

Because any homomorphism of $S$ into an inverse semigroup can be uniquely factored through $\eta, S$ can be. embedded in an inverse semigroup if and only if $\eta$ is one-to-one. We can use this to give a short proof of Schein's theorem [16] which gives necessary and sufficient conditions for embedding semigroups in inverse semigroups.

Let $S=S^{1}$ be a semigroup. Then a nonempty subset $H$ of $S$ is strong if 
$a x, b x, a y \in H$ together imply by $\in H$. Clearly, if nonvoid, the intersection of strong subsets is strong.

Let $H \neq \square$ be a strong subset of $S=S^{1}$ and define

$$
x \equiv y \quad\left(R_{H}\right) \quad \text { if and only if } H \cdot x=H \cdot y
$$

where, for example, $H^{\cdot} \cdot x=\{u \in S: x u \in H\}$. Then $R_{H}$ is a right congruence on on $S[2, \S 10.2]$ and can be used to construct a representation of $S$ by one-to-one partial transformations in the following way $[2, \$ 11.4]$. Set $W_{H}=\left\{x \in S: H \cdot{ }^{\circ} x=\square\right\}$. $W_{H}$ is clearly an $R_{H}$-class of $S$, and let $X_{H}$ be the set of $R_{H}$-classes different from $W_{H}$. For each $a \in S$, define

$$
\bar{x} \rho_{a}^{H}=\overline{x a} \text { for each } \bar{x} \in X_{H} \text { such that } \overline{x a} \in X_{H} \text {. }
$$

Then the mapping $\rho^{H}: a \rightarrow \rho_{a}^{H}$ is a representation of $S$ by one-to-one partial transformations of $X_{H}$; thus $\rho^{H}$ is a homomorphism of $S$ into the symmetric inverse semigroup $g\left(X_{H}\right)$ on $X_{H}$.

Recall that, if $T$ is an inverse semigroup, the natural partial order on $T$ is defined by

$$
x \leq y \text { if and only if } x=e y \text { for some } e=e^{2} \in T[2, \$ 7.1]
$$

Lemma 1.1. Let $\theta$ be a bomomorphism of a semigroup $S=S^{1}$ into an inverse semigroup $T$ and let $a \in S$. Then $K=\{x \in S: a \theta \leq x \theta\}$ is a strong subset of $S$ which contains $a$.

Proof. Suppose $b x, b y, c x \in K$. Then $a \theta \leq(b x) \theta, a \theta \leq(b y) \theta, a \theta \leq(c x) \theta$ and so, also, $(a \theta)^{-1} \leq(b x) \theta^{-1}$. Thus

$$
a \theta=a \theta(a \theta)^{-1} a \theta \leq(c x) \theta(b x) \theta^{-1}(b y) \theta=c \theta\left(x \theta x \theta^{-1} b \theta^{-1} b \theta\right) y \theta \leq(c y) \theta .
$$

Hence $c y \in K$. This shows that $K$ is strong and, clearly, $a \in K$.

Lemma 1.2. Let $S=S^{1}$ be a semigroup and let $a \in S$. Then $\hat{a}=$ $\{x \in S: a \eta \leq x \eta\}$ is the smallest strong subset of $S$ wbicb contains a.

Proof. By Lemma 1.1, $\hat{a}$ is a strong subset of $S$ which contains $a$. On the other hand, suppose that $H$ is a strong subset of $S$ and $a \in H$. Let $\rho^{H}: S \rightarrow g\left(x_{H}\right)$ be the representation of $S$ obtained from $H$ and suppose that $x \in \hat{a}$. Since $\rho^{H}$ can be factored through $\eta$, it follows that $a \rho^{H} \leq x \rho^{H}$ and so, in particular, the domain $\Delta \rho_{a}^{H}$ of $\rho_{a}^{H}$ is contained in $\Delta \rho_{x}^{H}$. Now $\bar{a}=\overline{1} \bar{a} \in X_{H}$ so $\bar{I} \in \Delta \rho_{a}^{H}$; hence $\overline{1} \in \Delta \rho_{x}^{H}$. Further, since $\rho_{a}^{H} \leq \rho_{x}^{H}$,

$$
\bar{a}=\overline{1} \rho_{a}^{H}=\overline{1} \rho_{x}^{H}=\bar{x} .
$$

Hence $H^{\cdot} \cdot x=H^{\circ} a$ and so, since $1 \in H^{\cdot} a, x \in H$. This shows that $\hat{a} \subseteq H$. 
Theorem 1.3 (Schein [16]). Let $S=S^{1}$ be a semigroup. Then $S$ can be embedded in an inverse semigroup if and only if for eacb pair of distinct elements of $S$ there is a strong subset of $S$ which contains one of the pair but not the other.

Proof. Suppose that $\eta$ is one-to-one and that $a \neq b$ in $S$. Then $a \eta \neq b \eta$ and so $a \eta \unlhd b \eta$ or $b \eta \unlhd a \eta$; thus $b \notin \hat{a}$ or $a \notin \hat{b}$.

Conversely, if $H$ is strong and $a \in H, b \notin H$ then, since $\hat{a} \subseteq H, b \notin \hat{a}$ and so $a \eta ₫ b \eta$; in particular, $a \eta \neq b \eta$.

The method of proof of Theorem 1.3 can be used to give the relationship between the ideal structure of $S$ and that of $I(S)$.

Proposition 1.4. Let $S=S^{1}$ be a semigroup and let $\eta: S \rightarrow I(S)$ be the canonical bomomorphism of $S$ into the free inverse semigroup on $S$. Then $a \eta(a \eta)^{-1}$ $\leq b \eta(b \eta)^{-1}$ if and only if $\hat{a} \cap b S \neq \square$.

Proof. Suppose $\hat{a} \cap b S \neq \square$. Then $b x \in \hat{a}$ for some $x \in S$ and so $a \eta \leq(b x) \eta$. Hence $a \eta=b \eta(b \eta)^{-1} a \eta$; that is $a \eta(a \eta)^{-1} \leq b \eta(b \eta)^{-1}$.

Conversely, suppose that $a \eta(a \eta)^{-1} \leq b \eta(b \eta)^{-1}$ and let $\rho$ be the representation of $S$ by one-to-one partial transformations obtained from the strong subset $\hat{a}$. Then, since $\rho$ can be factored through $\eta, a \rho(a \rho)^{-1} \leq b \rho(b \rho)^{-1}$; that is $\Delta a \rho \subseteq \Delta b \rho$. Since $\bar{I} \in \Delta a \rho$, this implies $\overline{1} \in \Delta \rho_{b}$ so that $\bar{b} \in \mathfrak{X}_{\hat{a}}$; that is $b S \cap \hat{a} \neq \square$.

Corollary 1.5. The mapping a defined by $(a S) \alpha=(a \eta) I(S)$ is an order isomorphism of the set of principal right ideals of $S$ into the set of principal right ideals of $I(S)$ if and only if $\hat{a} \cap b S \neq \square$ implies $a \in b S$.

If $T$ is an inverse semigroup, then the intersection of principal right (left) ideals is again principal and, indeed, if $a T \cap b T=c T$ then $x a T \cap x b T=x c T$ for each $x \in T$. Thus, when one considers the relationships between $S$ and $I(S)$ it is of interest to suppose that $S$ is naturally quasisemilatticed in the sense of the following definition.

Definition. Let $S=S^{1}$ be a semigroup. Then $S$ is naturally quasisemilatticed if, for each $a, b \in S$, there exists $a \wedge, b \in S$ such that $a S \cap b S=(a \wedge, b) S$ and, for each $x \in S,\left(x a \wedge_{r} x b\right) S=x(a \wedge, b) S$ and dually for left ideals.

If $S=S^{1}$ is a semigroup in which $\mathfrak{D}$ is trivial then $S$ is naturally quasisemilatticed if and only if it is a left semilatticed semigroup under the partial ordering $a \leq r b$ if and only if $a \in b S$ and dually. Any semigroup in which the sets of principal left and right ideals form chains under inclusion is naturally quasisemilatticed as is the positive cone of an $l$-group and the multiplicative semigroup of a principal ideal domain. The free monoid on a set $X$ is not naturally quasisemilatticed; however if a zero is adjoined, the resulting monoid is naturally quasisemilatticed. 
In $\$ 6$ we shall give necessary and sufficient conditions for embedding a naturally quasisemilatticed semigroup into an inverse semigroup. These conditions, unlike those in Theorem 1.3, do not involve strong subsets; the latter are hard to find in general.

2. Shift representations of semigroups. Let $S=S^{1}$ be a semigroup and let $\sigma$ be an equivalence on $S \times S$ which obeys the foll owing condition:

$$
(a, x b) \sigma(c, x d) \text { if and only if }(a x, b) \sigma(c x, d)
$$

for all $a, b, c, d, x \in S$ and, for each $x \in S$, define a partial transformation $\rho_{x}^{\sigma}$ on the set $(S \times S) / \sigma$ of $\sigma$-classes by

$$
(a, x b) \sigma \rho_{x}^{\sigma}=(a x, b) \sigma .
$$

Then $\rho_{x}^{\sigma}$ is clearly a one-to-one partial transformation of $(S \times S) / \sigma$.

Lemma 2.1. Let $\sigma$ be an equivalence, whicb obeys (1), on a semigroup $S=S^{1}$. Then the mapping $\rho^{\sigma}: S \rightarrow I((S \times S) / \sigma)$ defined by $x \rho^{\sigma}=\rho_{x}^{\sigma}$ is a representation of $S$ by one-to-one partial transformations $(S \times S) / \sigma$ if and only if

$$
(a, b) \sigma(c, d) \text { implies }(a, b) \sigma(x a, d y) \text { for some } x, y \in S \text {. }
$$

Proof. For any $a, b \in S, \Delta \rho_{a b}^{\sigma} \subseteq \Delta \rho_{a}^{\sigma} \rho_{b}^{\sigma}$ and further, if $(x, a b y) \sigma \in \Delta \rho_{a b}^{\sigma}$,

$$
(x, a b y) \sigma \rho_{a b}^{\sigma}=(x a b, y) \sigma=(x a, b y) \sigma \rho_{b}^{\sigma}=(x, a b y) \sigma \rho_{a}^{\sigma} \rho_{b}^{\sigma} .
$$

Hence $\rho^{\sigma}$ is a representation if and only if $\Delta \rho_{a}^{\sigma} \rho_{b}^{\sigma} \subseteq \Delta \rho_{a b}^{\sigma}$ for all $a, b \in S$.

Suppose that (2) holds. Then $(x, a y) \sigma \in \Delta \rho_{a}^{\sigma} \rho_{b}^{\sigma}$ implies $(x a, y) \sigma(u, b v)$ for some $u, v \in S$. Hence, by (2), $(x a, y) \sigma(r x a, b v s)$ for some $r, s \in S$. Thus, by (1), $(x, a y) \sigma(r x, a b v s)$ so that $(x, a y) \sigma \in \Delta \rho_{a b}^{\sigma}$.

Conversely, suppose that $\Delta \rho_{a}^{\sigma} \rho_{b}^{\sigma} \subseteq \Delta \rho_{a b}^{\sigma}$ and let $(a, b) \sigma(c, d)$. Then $(1, a b) \sigma \rho_{a}^{\sigma}=(a, b) \sigma=(c, d) \sigma$ implies $(1, a b) \sigma \in \Delta \rho_{a}^{\sigma} \rho_{d}^{\sigma}=\Delta \rho_{a d}^{\sigma}$. Hence $(1, a b) \sigma$ $(x, a d y)$ for some $x, y \in S$ and so, by $(1),(a, b) \sigma(x a, d y)$.

Definition. If $S=S^{1}$ is a semigroup then an equivalence $\sigma$ on $S \times S$ is called a shift equivalence if (1) and (2) are satisfied. If $\sigma$ is a shift equivalence on $S \times S$ then the corresponding representation $\rho^{\sigma}$ of $S$ by one-to-one partial transformations of $(S \times S) / \sigma$ is called a shift representation of $S$.

Equivalence relations on $S \times S$ which obey (1) arise naturally when one considers homomorphisms of $S$ into inverse semigroups as the following examples show.

Proposition 2.2. Let $\theta$ be a bomomorphism of a semigroup $S=S^{1}$ into an inverse semigroup $T$ and define equivalences $\sigma_{L}, \sigma_{R}, \sigma_{E}$ on $S \times S$ as follows: 


$$
\begin{aligned}
& (a, b) \sigma_{L}(c, d) \Leftrightarrow b \theta(a b) \theta^{-1}=d \theta(c d) \theta^{-1}, \\
& (a, b) \sigma_{R}(c, d) \Leftrightarrow(a b) \theta^{-1} a \theta=(c d) \theta^{-1} c \theta, \\
& (a, b) \sigma_{E}(c, d) \Leftrightarrow a \theta^{-1} a \theta b \theta b \theta^{-1}=c \theta^{-1} c \theta d \theta d \theta^{-1} .
\end{aligned}
$$

Then eacb of these equivalences obeys (1).

Proof. We show $\sigma_{E}$ obeys (1).

$$
\begin{aligned}
(a, x b) \sigma_{E}(c, x d) & \Leftrightarrow a \theta^{-1} a \theta(x b)(x b) \theta^{-1}=c \theta^{-1} c \theta(x d) \theta(x d) \theta^{-1} \\
& \Leftrightarrow a \theta^{-1}(a x) \theta b \theta b \theta^{-1} x \theta^{-1}=c \theta^{-1}(c x) \theta d \theta d \theta^{-1} x \theta^{-1} \\
& \Leftrightarrow x \theta^{-1} a \theta^{-1}(a x) \cdot \theta b \theta b \theta^{-1}=x \theta^{-1} c \theta^{-1}(c x) \theta d \theta d \theta^{-1} \\
& \Leftrightarrow(a x) \theta^{-1}(a x) \theta b \theta b \theta^{-1}=(c x) \theta^{-1}(c x) \theta d \theta d \theta^{-1} \\
& \Leftrightarrow(a x, b) \sigma_{E}(c x, d)
\end{aligned}
$$

since idempotents commute.

The other two are proved similarly.

There is clearly a smallest equivalence on $S \times S$ which obeys (1). In some important cases, this can easily be described and is a shift equivalence.

Lemma 2.3. Let $S=S^{1}$ be a semigroup and define a relation $\tau_{0}$ on $S \times S$ by $(a, b) \tau_{0}(c, d) \Leftrightarrow$ there exist $x_{0}, \cdots, x_{n^{\prime}} y_{0}, \ldots, y_{n}$ sucb that $a=x_{0^{\circ}} c=x_{n^{\prime}}$ $b=y_{0}, d=y_{n}$ and $x_{i-1} y_{i-1}=x_{i} y_{i-1}=x_{i} y_{i}, 1 \leq i \leq n$. Then $\tau_{0}$ is an equivalence and is contained in the smallest equivalence on $S \times S$ wbicb obeys (1).

Proof. $\tau_{0}$ is clearly an equivalence on $S \times S$. Further, if $\sigma$ is an equivalence on $S \times S$ which obeys (1) then $x_{i-1} y_{i-1}=x_{i} y_{i-1}=x_{i} y_{i}$ implies

$$
\left(x_{i-1} y_{i-1}, 1\right) \sigma\left(x_{i} y_{i-1}, 1\right) \text { and }\left(1, x_{i} y_{i-1}\right) \sigma\left(1, x_{i} y_{i}\right) \text {. }
$$

Thus, by (1), $\left(x_{i-1}, y_{i-1}\right) \sigma\left(x_{i}, y_{i-1}\right) \sigma\left(x_{i}, y_{i}\right)$ so that, from the definition of $\tau_{0}, \tau_{0} \subseteq \sigma$.

Propositions 2.6, 2.7, 2.9 give examples of types of semigroups on which $\tau_{0}$ is a shift and thus is the finest shift on $S \times S$. Under these circumstances we can use $\tau_{0}$ to give necessary and sufficient conditions for embeddability in inverse semigroups.

Lemma 2.4. Let $S=S^{1}$ be a semigroup such that $\tau_{0}$ is a shift and let $\rho$ be the shift representation associated with $\tau_{0}$. Then $\rho_{a}=\rho_{b}$ if and only if $\hat{a}=\hat{b}$.

Proof. If $\tau_{0}$ is a shift, then $\rho$ can be factored through $\eta$ and so $\hat{a}=\hat{b}$ implies $\rho_{a}=\rho_{b} \cdot$ 
On the other hand, $\rho_{a}=\rho_{b}$ implies $(1, a) \tau_{0}(x, b y)$ and $(a, 1) \tau_{0}(x b, y)$ for some $x, y \in S$. The first of these equivalences implies the existence of $u_{0}, \cdots$, $u_{n^{\prime}} v_{0}, \cdots, v_{n}$ in $S$ such that $u_{0}=1, u_{n_{n}}=x, v_{0}=a, v_{n}=b y$ and $u_{i-1} v_{i-1}=$ $u_{i} v_{i-1}=u_{i} v_{i}, 1 \leq i \leq n$. Then $v_{0}=a \in \hat{a}$. Suppose $v_{i-1} \in \hat{a} ;$ then $u_{i-1} v_{i-1}=$ $u_{i} v_{i-1}=u_{i} v_{i}=a \in \hat{a}$ implies $u_{i-1} v_{i} \in \hat{a}$ and so $u_{i-1} \epsilon \hat{a} \cdot v_{i} \cap \hat{a} \cdot v_{i-1}$. Since $\hat{a}$ is strong and $1 \in \hat{a} \cdot v_{i-1}$, this implies $1 \in \hat{a} \cdot v_{i}$ so that $v_{i} \in \hat{a}$. Hence, by induction, by $\in \hat{a}$. Dually, the second equivalence implies $x \hat{b} \in \hat{a}$.

Since $x b y=a \in \hat{a}$ and $b y \in \hat{a}$ we have $y \in \hat{a} \cdot x b \cap \hat{a} \cdot b$ and so, since $\hat{a}$ is strong and $1, \in \hat{a}^{\cdot} \cdot x b, 1 \in \hat{a} \cdot b$; thus $b \in \hat{a}$. Finally, by duality, we also get $a \in \hat{b}$. Hence $\hat{a}=\hat{b}$.

Theorem 2.5. Let $S=S^{1}$ be a semigroup on which $\tau_{0}$ is a shift and let $\rho: S \rightarrow g\left((S \times S) / \tau_{0}\right)$ be the corresponding shift representation. Then $S$ can be embedded in an inverse semigroup if and only if $\rho$ is one-to-one.

We now give some examples of semigroups in which $\tau_{0}$ obeys (1) and (2).

Proposition 2.6. Let $S=S^{1}$ be a left cancellative semigroup. Then $\tau_{0}$ is a shift equivalence on $S \times S$.

Proof. Suppose $S$ is left cancellative and let $(a, b) \tau_{0}(c, d)$. Then $a=x_{0}$, $c=x_{n}, b=y_{0}, d=y_{n}$ and $x_{i-\mathrm{i}} y_{i-1}=x_{i} y_{i-1}=x_{i} y_{i}, 1 \leq i \leq n$, for some $x_{i}, y_{i} \in S$. Since $S$ is left cancellative, this implies $y_{i-1}=y_{i}, 1 \leq i \leq n$; hence each $y_{i}$ is $b$ and so $(a, b) \tau_{0}(c, d)$ implies $b=d$ and $a b=c b$. On the other hand, $b=d, a b=c b$ clearly implies $(a, b) \tau_{0}(c, d)$. Hence

$$
(a, b) \tau_{0}(c, d) \Leftrightarrow b=d, \quad a b=c b .
$$

It follows from this characterisation of $\tau_{0}$ that $(a, x b) \tau_{0}(c, x d)$ if and only if $a x b=c x d, x b=x d$. Since $S$ is.left cancellative, the last two equations hold if and only if $a x b=c x d$ and $b=d$. Hence (1) holds. Finally, from the characterisation of $\tau_{0},(a, b) \tau_{0}(c, d)$ implies $(a, b) \tau_{0}(a, d)$ so that (2) holds trivially.

Proposition 2.7. Let $S=S^{1}$ be an inverse semigroup. Then $\tau_{0}$ is a sbift equivalence on $S \times S$.

Proof. Suppose $(a, x b) \tau_{0}(c, x d)$; then $a=u_{0}, c=u_{n}, x b=v_{0}, x d=v_{n}$ and $u_{i-1} v_{i-1}=u_{i} v_{i-1}=u_{i} v_{i}, 1 \leq i \leq n$, for some $u_{i}, v_{i} \in S$. Set $p_{0}=a x, p_{n}=c x$, $q_{0}=b, q_{n}=d$ and $p_{i}=u_{i} x, q_{i}=x^{-1} v_{i}, 1<i<n$. We show that $p_{i-1} q_{i-1}=$ $p_{i} q_{i-1}=p_{i} q_{i}, 1 \leq i \leq n$. This proves that $(a x, b) \tau_{0}(c x, d)$ and, together $w i t h$ its dual, gives (1).

Since $u_{i-1} v_{i-1}=u_{i} v_{i-1}$, it follows that $u_{i-1} v_{i-1} v_{i-1}^{-1} x x^{-1}=u_{i} v_{i-1} v_{i-1}^{-1} x x^{-1}$ and so, since idempotents commute, $\left(u_{i-1} x\right)\left(x^{-1} v_{i-1}\right)=\left(u_{i} x\right)\left(x^{-1} v_{i-1}\right)$; 
similarly $\left(u_{i} x\right)\left(x^{-1} v_{i-1}\right)=\left(u_{i} x\right)\left(x^{-1} v_{i}\right), 1 \leq i \leq n$. Hence, for $1<i<n, p_{i-1} q_{i-1}$ $=p_{i} q_{i-1}=p_{i} q_{i} \cdot$ Further

$$
p_{0} q_{0}=a \times b=u_{0} v_{0}=u_{1} v_{0}=u_{1} x b=p_{1} q_{0}
$$

and, as above, $u_{1} x x^{-1} v_{0}=u_{1} x x^{-1} v_{1}=p_{1} q_{1}$ so that, since $v_{0}=x b, p_{1} q_{0}=u_{1} v_{0}$ $=u_{1} x x^{-1} v_{0}=p_{1} q_{1}$. Similarly $p_{n-1} q_{n-1}=p_{n} q_{n-1}=p_{n} q_{n}$. Thus $p_{i-1} q_{i-1}=$ $p_{i} q_{i-1}=p_{i} q_{i}, 1 \leq i \leq n$.

Finally, suppose that $(a, b) \tau_{0}(c, d)$; then $a=x_{0}, c=x_{n}, b=y_{0}, d=y_{n}$ and $x_{i-1} y_{i-1}=x_{i} y_{i-1}=x_{i} y_{i}, 1 \leq i \leq n$, for some $x_{i}, y_{i} \in S$ and some positive integer $n$. As in the immediately preceding paragraph, this implies $\left(x_{0} a^{-1} a, y_{0}\right)$ $\tau_{0}\left(x_{n} a^{-1} a, y_{n}\right) ;$ that is $(a, b) \tau_{0}\left(c a^{-1} a_{0} d\right)$. Hence (2) hol ds.

Corollary 2.8. Let $S=S^{1}$ be an inverse semigroup and let $\rho$ be the shift representation associated with $\tau_{0}$. Then $\rho$ is faithful.

Proposition 2.9. Let $S=S^{1}$ be a naturally quasiordered semigroup on which $D$ is trivial. Then $\tau_{0}$ is a shift equivalence on $S \times S$.

Proof. This is a special case of Theorem 3.9 so we omit a proof.

\section{Fundamental inverse semigroups separated over a semigroup $S$.}

Lemma 3.1. Let $\theta$ be a homomorphism of a semigroup $S$ into an inverse semigroup $T$. Let $a, b, c \in S$ and suppose that

$$
a \theta a \theta^{-1} b \theta b \theta^{-1}=x \theta x \theta^{-1}, \quad b \theta^{-1} b \theta c \theta^{-1} c \theta=u \theta^{-1} u \theta
$$

where $x=a y=b z, u=v b=w c$. Then

$$
a \theta^{-1} b \theta c \theta^{-1}=y \theta(v b z) \theta^{-1} w \theta .
$$

Proof. For convenience of notation, let us identify $S$ with its image in $T$. Then

$$
\begin{aligned}
a^{-1} b c^{-1} & =a^{-1} a a^{-1} b b^{-1} b c^{-1}=a^{-1}(a y)(a y)^{-1} b c^{-1}=a^{-1} a y y^{-1} a^{-1} b c^{-1} \\
= & y y^{-1} a^{-1} b c^{-1}=y x^{-1} b c^{-1}=y x^{-1} b b^{-1} b c^{-1} c c^{-1}=y x^{-1} b(w c)^{-1} u c c^{-1} \\
& =y x^{-1} b(w c)^{-1} w=y(b z)^{-1} b(v b)^{-1} w=y(v b z)^{-1} w
\end{aligned}
$$

since idempotents in $T$ commute.

Lemma 3.1 is similar to Lemma 3.4 in [5].

Theorem 3.2. Let $\theta$ be a bomomorphism of $S=S^{1}$ into an inverse semigroup $T$. If $T$ is separated over $S$ by $\theta$ then $T=\left\{a \theta b \theta^{-1} c \theta: b \in S a \cap c S, a, c \in S\right\}$. 
Proof. As in Lemma 3.1, we identify $S$ and $S \theta$. Let $a b^{-1} c, d e^{-1} f \in K$, where $K$ denotes the right side of the equation for $T$, and suppose that $b=u a=c v$, $e=p d=f q$.

By Lemma 2.1, if $b b^{-1} c d(c d)^{-1}=b b^{-1}$ and $(c d)^{-1} c d e^{-1} e=k^{-1} k$ with $b=b y=c d z$ and $k=x c d=w e$, then

$$
b^{-1} c d e^{-1}=y(x c d z)^{-1} w
$$

so that $a b^{-1} c d e^{-1} f=a y(x c d z)^{-1} w f$. Further $x c d z=x b y=x u a y \in S a y$ and $x c d z=w e z=w f q z \in w f S$ so that $a b^{-1} c d e^{-1} f \in K$. Since, by Lemma 3.1, $K$ is closed under inverses, it follows that $K=T$.

Definition. Let $T$ be an inverse semigroup and let $S=S^{1}$ be a subsemigroup of $T$. Then $T$ is an inverse semigroup of strong quotients of $S$ if each element of $T$ is of the form $a b^{-1} c$ where $b \in S a \cap c S$.

In the light of this definition, we have

Corollary 3.3. Let $T$ be an inverse semigroup which is separated over a sub. semigroup $S$. Then $T$ is an inverse semigroup of strong quotients of $S$.

The inverse semigroups which are separated over a semigroup $S=S^{1}$ appear to be closely related to the shift representations of $S$. We have not been able to determine this relationship in general; however we have been able to characterise fundamental inverse semigroups which are separated over $S$.

Lemma 3.4. Let $\theta$ be a bomomorphism of a semigroup $S=S^{1}$ into an inverse semigroup $T$. Suppose that $T$ is separated over $S$ by $\theta$ and define $\sigma_{E}$ on $S \times S$ by

$$
(a, b) \sigma_{E}(c, d) \Leftrightarrow a \theta^{-1} a \theta b \theta b \theta^{-1}=c \theta^{-1} c \theta d \theta d \theta^{-1}
$$

for all $a, b, c, d \in S$. Then $\sigma_{E}$ is a shift equivalence on $S \times S$ and $S \times S / \sigma_{E}$ is a semilattice, isomorphic to the semilattice of idempotents of $T$, under the partial ordering

$$
(a, b) \sigma_{E} \leq(c, d) \sigma_{E} \Leftrightarrow(a, b) \sigma_{E}(u, v) \text { for some } u \in S a \cap S c, v \in b S \cap d S .
$$

Proof. Since $T$ is separated over $S$, Theorem 3.2 shows that each element of $T$ is of the form $a \theta b \theta^{-1} c \theta$ where $b \in S a \cap c S$. For such an element of $T$,

$$
\begin{aligned}
a \theta b \theta^{-1} c \theta\left(a \theta b \theta^{-1} c \theta\right)^{-1} & =a \theta b \theta^{-1} c \theta c \theta^{-1} b \theta a \theta^{-1} \\
& =a \theta b \theta^{-1} b \theta a \theta^{-1} \quad \text { since } b \in c S \\
& =u \theta^{-1} u \theta a \theta a \theta^{-1} \quad \text { if } b=u a .
\end{aligned}
$$

Hence the mapping defined by $(u, a) \sigma_{E} \rightarrow u \theta^{-1} u \theta a \theta a \theta^{-1}$ is a bijection of $(S \times S) / \sigma_{E}$ onto the semilattice of idempotents of $T$. Further, since 
$a \theta^{-1} a \theta b \theta b \theta^{-1} \leq c \theta^{-1} c \theta d \theta d \theta^{-1}$ if and only if $a \theta^{-1} a \theta b \theta b \theta^{-1}$ -

$a \theta^{-1} a \theta c \theta^{-1} c \theta b \theta b \theta^{-1} d \theta d \theta^{-1}$ and since $T$ is separated over $S, a \theta^{-1} a \theta b \theta b \theta^{-1} \leq$ $c \theta^{-1} c \theta d \theta d \theta^{-1}$ if and only if $(a, b) \sigma_{E}(u, v)$ for some $u \in S a \cap S c, v \in b S \cap d S$. Hence $(S \times S) / \sigma_{E}$ is a semilattice under

$(a, b) \sigma_{E} \leq(c, d) \sigma_{E} \Leftrightarrow(a, b) \sigma_{E}(u, v)$ for some $u \in S a \cap S c, v \in b S \cap d S$

Finally, Proposition 2.2 shows that $\sigma_{E}$ obeys (1) while, since $(S \times S) / \sigma_{E}$ is a semilattice under the partial order described above, $\sigma_{E}$ clearly obeys (2). Hence $\sigma_{E}$ is a shift.

Lemma 3.5. Let $S=S^{1}$ be a semigroup and let $\sigma$ be an equivalence on $S \times S$. Suppose that $(S \times S) / \sigma$ is a semilattice under

$$
(a, b) \sigma \leq(c, d) \sigma \Leftrightarrow(a, b) \sigma(u, v) \text { for some } u \in S a \cap S c, v \in b S \cap d S,
$$

Then,

(i) $(1, a)_{\sigma} \wedge(1, b)_{\sigma}=(1, v)_{\sigma}$ for some $v \in a S \cap b S$,

(ii) $(a, 1)_{\sigma} \wedge(b, 1) \sigma=(u, 1) \sigma$ for some $u \in S a \cap S b$,

(iii) $(a, 1)_{\sigma} \wedge(1, b)_{\sigma}=(a, b)_{\sigma}$

for $a, b \in S$.

Proof. (i) Suppose $(1, a)_{\sigma} \wedge(1, b)_{\sigma}=(x, y)_{\sigma}$. Then, because $(x, y) \sigma \leq(1, a) \sigma$, there exist $x_{1} \in S, y_{1} \in y S \cap a S$ such that $\left(x_{1}, y_{1}\right) \sigma(x, y)$. Since $\left(x_{1}, y_{1}\right) \sigma \leq$ $(1, b)_{\sigma}$, there exist $u \in S, v \in y_{1} S \cap b S \subseteq a S \cap b S$ such that $\left(x_{1}, y_{1}\right) \sigma(u, v)$. Thus $(1, a)_{\sigma} \wedge(1, b)_{\sigma}=(u, v)_{\sigma}$. But $(u, v)_{\sigma} \leq(1, v)_{\sigma} \leq(1, a)_{\sigma},(1, b)_{\sigma}$ from the definition of $\leq$ since $v \in a S \cap b S$. Hence we must have $(1, a) \sigma \wedge(1, b) \sigma=(1, v) \sigma$.

(ii) This is dual to (i).

(iii) From the definition of the partial order on $(S \times S) / \sigma,(a, b) \sigma \leq(a, 1) \sigma$, $(1, b) \sigma$. On the other hand, if $(x, y)_{\sigma} \leq(a, 1) \sigma,(1, b)_{\sigma}$, then $(x, y) \sigma\left(x_{1}, y_{1}\right)$ for some $x_{1} \in S a \cap S x$ and then, since $\left(x_{1}, y_{1}\right) \sigma \leq(1, b)_{\sigma},\left(x_{1} y_{1}\right) \sigma\left(x_{2}, y_{2}\right)$ for some $x_{2} \in S x_{1} \cap S a$ and $y_{2} \in y_{1} S \cap b S \subseteq b S$. Thus $(x, y)_{\sigma}=\left(x_{2}, y_{2}\right)_{\sigma} \leq(a, b)_{\sigma}$. Hence $(a, 1)_{\sigma} \wedge(1, b)_{\sigma}=(a, b) \sigma$.

Suppose that $T$ is an inverse semigroup with semilattice of idempotents $E$ and for each $a \in T$ define a partial transformation $\mu_{a}$ of $E$ by $x \mu_{a}=a^{-1} x a$ for each $x \in E a a^{-1}$. Then Munn [11] shows that $\mu: T \rightarrow g(E)$ defined by $a \mu=\mu_{a}$ is a representation of $T$ by partial one-to-one transformations of $E$ and that $T / \mu$ "is" the maximum fundamental homomorphic image of $T$.

Theorem 3.6. Let $S=S^{1}$ be a semigroup and let $\theta$ be a bomeomorphism of $S$ into a fundamental inverse semigroup $T$ which is separated over $S$ by $\theta$. Define $\sigma_{E}$ on $S \times S$ by 


$$
(a, b) \sigma_{E}(c, d) \Leftrightarrow a \theta^{-1} a \theta b \theta b \theta^{-1}=c \theta^{-1} c \theta d \theta d \theta^{-1}
$$

and let $\rho: S \rightarrow g\left((S \times S) / \sigma_{E}\right)$ be the shift representation associated with $\sigma_{E^{*}}$. Then $T$ is isomorphic to the inverse bull of $S \rho$ in $g\left((S \times S) / \sigma_{E}\right)$.

Conversely, let $\sigma$ be an equivalence on $S \times S$ which obeys (1) and is sucb that $(S \times S) / \sigma$ is a semilattice under

$$
(a, b) \sigma \leq(c, d) \sigma \Leftrightarrow(a, b) \sigma(u, v) \text { for some } u \in S a \cap S c, v \in b S \cap d S
$$

and let $\rho$ be the shift representation associated with $\sigma$. Then the inverse bull of $S \rho$ in $g((S \times S) / \sigma)$ is fundamental and $\sigma=\sigma_{E}$.

Proof. Let $\theta$ be as in the statement of the theorem. Then, by Lemma 3.4, the mapping $\phi$ defined by $a \phi=(a, b) \sigma_{E}$ if $a=a \theta^{-1} a \theta b \theta b \theta^{-1}$ is an isomorphism from the set $E$ of idempotents of $T$ onto $(S \times S) / \sigma_{E}$. Thus we can use $(S \times S) / \sigma_{E}$ to obtain a representation $\psi$ of $T$ equivalent to $\mu$ and hence to obtain an isomorphic copy of $T / \mu$. For each $\alpha \in T$, since $\psi$ is equivalent to $\mu$,

$$
\Delta \psi_{a}=\left\{e \phi \in(S \times S) / \sigma_{E}: e \in \Delta \mu_{a}\right\}=\left\{e \phi \in(S \times S) / \sigma_{E}: e \leq a a^{-1}\right\} \text {. }
$$

Hence, if $a=a \theta(b \theta)^{-1} c \theta$, where $b=u a=c v$,

$$
\begin{aligned}
\Delta \psi_{a} & =\left\{e \phi: e \leq a \theta(b \theta)^{-1} c \theta c \theta^{-1} b \theta a \theta^{-1}\right\} \\
& =\left\{e \phi: e \leq u \theta^{-1} u \theta a \theta a \theta^{-1}\right\}=\left\{e \phi: e \phi \leq(u, a) \sigma_{E}\right\} \\
& =\left\{(x u, a y) \sigma_{E}: x, y \in S\right\} \text { by Lemma 3.4. }
\end{aligned}
$$

This is independent of the particular choice of $a, b, c, u, v \in S$, with $b=u a=c v$, such that $a=a \theta(b \theta)^{-1} c \theta$. Further, using the fact that $\psi$ is equivalent to $\mu$, direct calculation shows that $(x u, a y) \sigma_{E} \psi_{a}=(x c, v y) \sigma_{E}$.

Consider the diagram

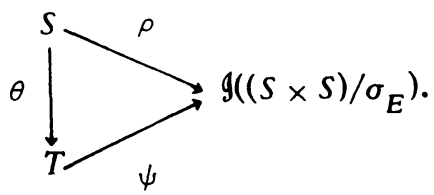

Let $a \in S$; then, since $a \theta=a \theta(a \theta)^{-1} a \theta$ where $a=1 \cdot a=a \cdot 1$,

$$
\Delta a \theta \psi=\left\{(x, a y) \sigma_{E}: x, y \in S\right\}=\Delta a \rho
$$

and, for $(x, a y) \sigma_{E} \in \Delta a \theta \psi$,

$$
(x, a y) \sigma_{E} a \theta \psi=(x a, y) \sigma_{E}=(x, a y) \sigma_{E} \rho_{a}
$$

from the calculations in the preceding paragraph. Hence $\rho=\theta \psi$ and the diagram commutes. Since $T \psi \approx T / \mu$ is generated, as an inverse semigroup, by $S \theta \psi=S \rho$, 
it follows that $T / \mu$ is isomorphic to the inverse hull of $S \rho$ in $9\left((S \times S) / \sigma_{E}\right)$. In particular, if $T$ is fundamental, so that $\mu$ is an isomorphism [11], $T$ is isomorphic to the inverse hull of $S \rho$ in $g\left((S \times S) / \sigma_{E}\right)$.

Conversely, suppose that $\sigma$ is an equivalence on $S \times S$ which obeys (1) and is such that $(S \times S) / \sigma$ is a semilattice under

$$
(a, b) \sigma \leq(c, d) \sigma \Leftrightarrow(a, b) \sigma(u, v) \quad \text { for some } u \in S_{a} \cap S_{c}, v \in b S \cap d S .
$$

Then, clearly, $\sigma$ obeys (2) and so gives rise to a shift representation $\rho$ of $S$ by one-to-one partial transformations of $(S \times S) / \sigma$. For each $a \in S$,

$$
\Delta \rho_{a}=\{(x, a y) \sigma: x, y \in S\}=\left\{(u, v)_{\sigma}:(u, v)_{\sigma} \leq(1, a) \sigma\right\}
$$

Hence, by Lemma 3.5 (i), since $(S \times S) / \sigma$ is a semilattice

$$
\begin{aligned}
\Delta \rho_{a} \cap \Delta \rho_{b} & =\left\{(u, v)_{\sigma:}(u, v)_{\sigma} \leq(1, a)_{\sigma} \wedge(1, b)_{\sigma}\right\} \\
& =\left\{(u, v)_{\sigma:}(u, v)_{\sigma} \leq(1, y)_{\sigma}\right\} \\
& =\Delta \rho_{y} \text { for some } y \in a S \cap b S .
\end{aligned}
$$

Thus $\rho_{a} \rho_{a}^{-1} \rho_{b} \rho_{b}^{-1}=\rho_{y} \rho_{y}^{-1}$ for some $y \in a S \cap b S$ and, dually, $\rho_{a}^{-1} \rho_{a} \rho_{b}^{-1} \rho_{b} \rho_{b}^{-1}=$ $\rho_{x}^{-1} \rho_{x}$ for some $x \in S a \cap S b$. Hence the inverse hull $K$ of $S \rho$ is separated over $S$ by $\rho$ and so, by Corollary 3.3, is an inverse semigroup of strong quotients of $S \rho$. In particular, the idempotents of $K$ are all of the form $\rho_{a}^{-1} \rho_{a} \rho_{b} \rho_{b}^{-1}$. Further,

$$
\rho_{a}^{-1} \rho_{a} \rho_{b} \rho_{b}^{-1} \leq \rho_{c}^{-1} \rho_{c} \rho_{d} \rho_{d}^{-1} \Leftrightarrow(a, b)_{\sigma} \leq(c, d)_{\sigma}
$$

by Lemma 3.5 (iii). Hence the semilattice of idempotents of $K$ is isomorphic to $(S \times S) / \sigma$ and $\sigma=\sigma_{E} \cdot$ From the proof of the first part of the theorem, $K / \mu$, the maximum fundamental homomorphic image of $K$, is isomorphic to the inverse hull of $S \rho$ in $9((S \times S) / \sigma)$; that is, to $K$ itself. Hence $K$ is fundamental.

Remark. The proof of the first part of Theorem 3.6 shows the following: if $T$ is separated by $\theta$ over $S$ then $T / \mu$ is isomorphic to the inverse hull of $S \rho$ in $g\left((S \times S) / \sigma_{E}\right)$.

The second part of the theorem shows that if $\sigma$ is an equivalence on $S \times S$ which obeys (1) and is such that $(S \times S) / \sigma$ is a semilattice under the relation

$$
(a, b) \sigma \leq(c, d) \sigma \Leftrightarrow(a, b) \sigma(u, v) \text { for some } u \in S a \cap S_{c}, v \in b S \cap d S,
$$

then there is a homomorphism of $S$ into an inverse semigroup $T$ with semilattice $(S \times S) / \sigma$.

Theorem 3.6 characterises fundamental inverse semigroups which are separated over $S$ in terms of equivalences on $S \times S$. To end this section, we show how such equivalences can be obtained from equivalences on $S$.

If $\pi$ is a right congruence on $S=S^{1}$ then there is a natural action of $S$ on the set $S / \pi$ of equivalence classes as follows: 


$$
a \pi \cdot x=(a x) \pi \text { for all } a, x \in S .
$$

Dually, if $\pi$ is a left congruerce on $S$, then $S$ acts naturally on the left of $S / \pi$.

Let $\pi$ be a right congruence on $S$ such that $S / \pi$ is a semilattice. We say that $S$ acts naturally on the semilattice $S / \pi$ if

$$
(\bar{a} \wedge \bar{b}) \cdot x=\bar{a} \cdot x \wedge \bar{b} \cdot x
$$

for all $\bar{a}, \bar{b} \in S / \pi, x \in S$.

A dual definition holds for left congruences.

Lemma 3.7. Let $\sigma$ be an equivalence on $S \times S$ which obeys (1) and is such that $(S \times S) / \sigma$ is a semilattice under the partial ordering

$$
(a, b) \sigma \leq(c, d) \sigma \Leftrightarrow(a, b) \sigma(u, v) \text { for some } u \in S a \cap S c, v \in b S \cap d S
$$

and define

$$
a L b \Leftrightarrow(a, 1) \sigma(b, 1), \quad a R b \Leftrightarrow(1, a) \sigma(1, b) .
$$

Then $L$ is a right congruence on $S, S / L$ is a semilattice (with operation $\Lambda_{l}$ ) under

$$
a L \leq b L \Leftrightarrow a L u \text { for some } u \in S a \cap S b
$$

and $S$ acts naturally on $S / L$. Dual results bold for $R$. Further

$$
(a, b) \sigma(c, d) \Rightarrow a b L\left(a \wedge_{l} c\right) b R\left(a \wedge_{l} c\right)\left(b \wedge_{r} d\right) L c\left(b \wedge_{r} d\right) R c d
$$

where, for example, $a \Lambda_{l} c$ denotes any element of $S$ sucb that $\left(a \Lambda_{l} c\right) L=$ $\left(a L \wedge_{l} c L\right)$.

Proof. Let $\rho$ be the shift representation associated with $\sigma$. Then $(a, b) \sigma$ $(c, d)$ if and only if $\rho_{a}^{-1} \rho_{a} \rho_{b} \rho_{b}^{-1}=\rho_{c}^{-1} \rho_{c} \rho_{d} \rho_{d}^{-1}$. Hence $a L b$ implies $a \rho^{-1} a \rho=$ $b \rho^{-1} b \rho$ which, in turn, implies $(a x) \rho^{-1}(a x) \rho=(b x) \rho^{-1}(b x) \rho$; that is, ax $L b x$.

Thus $L$ is a right congruence on $S$.

Let $a, b \in S$ and pick $u \in S a \cap S b$ such that $(a, 1) \sigma \wedge(b, 1) \sigma=(u, 1) \sigma$; by Lemma 3.5 (iii) such an element exists. Then, from the definition of the partial order on $S / L, u L \leq a L, b L$. On the other hand, if $v L \leq a L, b L$ then $v L=y L$ for some $y \in S a \cap S b$ and so $(v, 1) \sigma=(y, 1) \sigma \leq(a, 1) \sigma,(b, 1) \sigma$; thus $(v, 1) \sigma \leq(u, 1) \sigma$. This implies $(v, 1) \sigma=(v, 1) \sigma \wedge(u, 1) \sigma$ and so, by Lemma 3.5 (iii), $(v, 1) \sigma=$ $(z, 1) \sigma$ for some $z \in S_{v} \cap S u \subseteq S u$. Hence $y L=z L \leq u L$. It follows that $S / L$ is a semilattice with $a L \wedge b L=u L$ where $u \in S a \cap S b$ is such that $(a, 1)_{\sigma} \wedge$ $(b, 1) \sigma=(u, 1) \sigma$. Further, $u \rho^{-1} u \rho=a \rho^{-1} a \rho b \rho^{-1} b \rho$ implies

$$
\begin{aligned}
(u x) \rho^{-1}(u x) \rho & =x \rho^{-1}\left(a \rho^{-1} a \rho b \rho^{-1} b \rho\right) x \rho \\
& =x \rho^{-1} a \rho^{-1} a \rho x \rho x \rho^{-1} b \rho^{-1} b \rho x \rho=(a x) \rho^{-1}(a x) \rho(b x) \rho^{-1}(b x) \rho .
\end{aligned}
$$


Hence $(u x) L=(a x) L \Lambda_{l}(b x) L$ and so $S$ acts naturally on $S / L$.

Next $(a, b) \sigma(c, d)$ if and only if

$a \rho^{-1} a \rho b \rho b \rho^{-1}=c \rho^{-1} c \rho d \rho d \rho^{-1}$

implies $a \rho^{-1} a \rho b \rho b \rho^{-1}=\left(a \wedge_{l} c\right) \rho^{-1}\left(a \wedge_{l} c\right) \rho b \rho b \rho^{-1}$

implies $\left(a \wedge_{l} c\right) \rho^{-1}\left(a \wedge_{l} c\right) \rho b \rho b \rho^{-1}=\left(a \wedge_{l} c\right) \rho^{-1}\left(a \wedge_{l} c\right) \rho\left(b \wedge_{r} d\right) \rho\left(b \wedge_{r} d\right) \rho^{-1}$

implies $\left(a \wedge_{l} c\right) \rho^{-1}\left(a \wedge_{l} c\right) \rho\left(b \wedge_{r} d\right) \rho\left(b \wedge_{r} d\right) \rho^{-1}=c \rho^{-1} c \rho\left(b \wedge_{r} d\right) \rho\left(b \wedge_{r} d\right) \rho^{-1}$

implies $c \rho^{-1} c \rho\left(b \wedge_{r} d\right) \rho\left(b \wedge_{r} d\right) \rho^{-1}=c \rho^{-1} c \rho d \rho d \rho^{-1}$

where, for example, $\left(a \Lambda_{l} c\right) L\left(a L \Lambda_{l} c L\right)$. These implications give in sequence

$(a b) \rho^{-1}(a b) \rho=\left[\left(a \wedge_{l} c\right) b\right] \rho^{-1}\left[\left(a \wedge_{l} c\right) b\right] \rho$ so $a b L\left(a \wedge_{l} c\right) b$

$\left[\left(a \wedge_{l} c\right) b\right] \rho\left[\left(a \wedge_{l} c\right) b\right] \rho^{-1}=\left[\left(a \wedge_{l} c\right)\left(b \wedge_{r} d\right)\right] \rho\left[\left(a \wedge_{l} c\right)\left(b \wedge_{r} d\right)\right] \rho^{-1}$

so $\left(a \wedge_{l} c\right) b R\left(a \wedge_{l} c\right)\left(b \wedge_{r} d\right)$

$\left[\left(a \wedge_{l} c\right)\left(b \wedge_{r} d\right)\right] \rho^{-1}\left[\left(a \wedge_{l} c\right)\left(b \wedge_{r} d\right)\right] \rho=\left[c\left(b \wedge_{r} d\right)\right] \rho^{-1}\left[c\left(b \wedge_{r} d\right)\right] \rho$

$$
\text { so }\left(a \wedge_{l} c\right)\left(b \wedge_{r} d\right) L c\left(b \wedge_{r} d\right)
$$

$\left[c\left(b \wedge_{r} d\right)\right] \rho\left[c\left(b \wedge_{r} d\right)\right] \rho^{-1}=(c d) \rho(c d) \rho^{-1}$ so $c\left(b \wedge_{r} d\right) R c d$

Hence $(a, b) \sigma(c, d)$ implies

$$
a b L\left(a \wedge_{l} c\right) b R\left(a \wedge_{l} c\right)\left(b \wedge_{r} d\right) L c\left(b \wedge_{r} d\right) R c d
$$

The converse follows, as in the proof of Theorem 3.8, because $\sigma$ is a shift.

Lemma 3.7 shows that $\sigma$ is determined by the equivalences $L$ and $R$. The next theorem shows how, starting with a pair of equivalences $L$ and $R$ we can obtain a shift $\sigma$.

Theorem 3.8. Let $S=S^{1}$ be a semigroup and let $L$ and $R$ be respectively right and left congruences on $S$ such that $S / L$ and $S / R$ are semilattices under

$$
\begin{aligned}
& a L \leq b L \Leftrightarrow a L c \text { for some } c \in S a \cap S b, \\
& a R \leq b R=a R c \text { for some } c \in a S \cap b S .
\end{aligned}
$$

Suppose also that $S$ acts naturally on the semilattices $S / L$ and $S / R$. Define a relation $\sigma=\sigma(L, R)$ on $S \times S$ by $(a, b) \sigma(c, d) \Leftrightarrow$ there exist finite sets $x_{0}, \ldots, x_{n}, y_{0}, \ldots, y_{n}$ in $S$ such that $a=x_{0}, c=x_{n}, b=y_{0}, d=y_{n}$ and, for $1 \leq i \leq n$,

$$
x_{i-1} y_{i-1} L x_{i} y_{i-1} R x_{i} y_{i}
$$


Then $\sigma$ is the finest equivalence on $S \times S$ with the following properties:

(i) $\sigma$ obeys (1),

(ii) $(S \times S) / \sigma$ is a semilattice under

$(a, b) / \sigma \leq(c, d) \sigma \Leftrightarrow(a, b) \sigma(u, v)$ for some $u \in S a \cap S c, v \in b S \cap d S$,

(iii) $a L c, b R d$ implies $(a, b) \sigma(c, d)$.

Proof. First, it is easy to see that $\sigma$ is an equivalence on $S \times S$. Suppose that $(a, b) \sigma(c, d)$ and let $u, v \in S$. Also let $x_{0}, \ldots, x_{n}, y_{0}, \ldots, y_{n}$ be as in the definition of $\sigma$. Then

$$
x_{i-1} y_{i-1} L x_{i} y_{i-1} \text { implies } x_{i-1} y_{i-1} \wedge_{l} u y_{i-1} L x_{i} y_{i-1} \wedge_{l} u y_{i-1}
$$

where, for $b, k \in S, b \wedge_{l} k$ denotes any element of $S b \cap S k$ such that $\left(b \wedge_{l} k\right) L=$ $b L \wedge_{l} k L$. Since $S$ acts naturally on the semilattice $S / L$, it follows from this that $\left(x_{i-1} \wedge_{l} u\right) y_{i-1} L\left(x_{i} \wedge_{l} u\right) y_{i-1}$ and hence, because $L$ is a right congruence, $\left(x_{i-1} \wedge_{l} u\right)\left(y_{i-1} \wedge_{r} v\right) L\left(x_{i} \wedge_{l} u\right)\left(y_{i-1} \wedge_{r} v\right)$. Similarly, $x_{i} y_{i-1} R x_{i} y_{i}$ implies $\left(x_{i} \wedge_{l} u\right)\left(y_{i-1} \wedge_{r} v\right) R\left(x_{i} \wedge_{l} u\right)\left(y_{i} \wedge_{r}^{*} v\right), \quad 1 \leq i \leq n$, Thus $\left(a \wedge_{l} u, b \wedge_{r} v\right) \sigma\left(c \wedge_{l} u, d \wedge_{r} v\right)$.

This shows, in particular, that the mapping $S / L \times S / R \rightarrow(S \times S) / \sigma$ defined by $(a L, b R) \rightarrow(a, b) \sigma$ is a semilattice homomorphism so that $(S \times S) / \sigma$ is a semilattice. Further, because of the order on $S / L$ and $S / R$,

$$
\begin{aligned}
(a, b) \sigma \leq(c, d) \sigma & \Leftrightarrow(a, b) \sigma\left(a \wedge_{l} c, b \wedge_{r} d\right) \\
& \Leftrightarrow(a, b) \sigma(u, v) \text { for some } u \in S a \cap S_{c}, v \in b S \cap d S .
\end{aligned}
$$

Suppose that $a=u_{0}, \cdots, u_{n}=c, x b=v_{0}, \cdots, v_{n}=x d$ and $u_{i-1} v_{i-1} L u_{i} v_{i-1} R u_{i} v_{i}$, $1 \leq i \leq n$. Define $q_{i}=w_{i}, 0 \leq 1 \leq n$, where $w_{i}$ is such that $x w_{i} \epsilon$ $x S \cap v_{i} S$ and $x w_{i} R=x R \wedge_{r} v_{i} R$ with $w_{0}=b, w_{n}=d$ and set $p_{i}=$ $u_{i} x, 0 \leq i \leq n$. Then

$$
p_{i-1} q_{i-1}=u_{i-1} x w_{i-1} L u_{i} x w_{i-1}=p_{i} q_{i-1} \text { for } 1<i \leq n
$$

since $x w_{i-1} \in v_{i-1} S$ and $L$ is a right congruence, and $p_{0} q_{0}=u_{0} x b=$ $u_{0} v_{0} L u_{1} v_{0}=u_{1} x b=p_{1} q_{0}$. Further, since $S$ acts naturally on the semilattice $S / R$,

$$
\begin{aligned}
p_{i} q_{i-1} R & =u_{i} x w_{i-1} R=u_{i} x R \wedge_{r} u_{i} v_{i-1} R \\
& =u_{i} x R \wedge_{r} u_{i} v_{i} R=u_{i}\left(x R \wedge_{r} v_{i} R\right) \\
& =u_{i} x w_{i} R=p_{i} q_{i} R, \quad 1 \leq i \leq n .
\end{aligned}
$$

Hence $(a x, b) \sigma(c x, d)$. The dual also holds so that $\sigma$ obeys (1).

Finally, $a L c, b R d$ implies $(a, 1) \sigma(c, 1)$ and $(1, b) \sigma(1, d)$ and so $\left(a \wedge_{l} 1, b \wedge_{r} 1\right) \sigma\left(c \wedge_{l} 1, d \wedge_{r} 1\right)$ by the first paragraph of the proof; thus $(a, c) \sigma(b, d)$ so that (iii) holds. 
Conversely, suppose that $\pi$ obeys (i), (ii), (iii). Then $x_{i-1} y_{i-1} L x_{i-1} y_{i-1} R x_{i} y_{i}$ implies $\left(x_{i-1} y_{i-1}, 1\right) \pi\left(x_{i} y_{i-1}, 1\right),\left(1, x_{i} y_{i-1}\right) \pi\left(1, x_{i} y_{i}\right)$ and so, by (i), $\left(x_{i-1}, y_{i-1}\right) \pi\left(x_{i}, y_{i-1}\right) \pi\left(x_{i}, y_{i}\right)$. Hence $(a, b) \sigma(c, d)$ implies $(a, b) \pi(c, d)$. Thus $\sigma$ is, in fact, the smallest equivalence on $S \times S$ which obeys (i) and (iii).

If $L$ and $R$ are right and left congruences on $S=S^{1}$, which obey the hypotheses of Theorem 3.7, it is easy to see that $\mathscr{C} \subseteq L, R \subseteq R$ where $\mathcal{L}$ and $R$ are the familiar Green's relations. Since $\mathcal{Q}$ and $\mathcal{R}$ obey the hypotheses of the theorem when $S$ is naturally quasisemilatticed we get, immediately, the following result which is of fundamental importance in later sections.

The orem 3.9. Let $S=S^{1}$ be a naturally quasisemilaticed semigroup and define a relation $\tau$ on $S \times S$ by

$$
(a, b) \tau(c, d) \Leftrightarrow \text { there exist finite sets } x_{0}, \ldots, x_{n}, y_{0}, \cdots, y_{n} \text { in } S
$$

sucb that $a=x_{0}, c=x_{n}, b=y_{0}, d=y_{n}$ and $x_{i-1} y_{i-1} \& x_{i} y_{i-1} R_{x_{i}} y_{i}, \quad 1 \leq i \leq n$. Then $\tau$ is the finest equivalence $\sigma$ on $S \times S$ which obeys (1) and is such that $(S \times S) / \sigma$ is a semilattice under

$$
(a, b) \sigma \leq(c, d) \sigma \Leftrightarrow(a, b) \sigma(u, v) \text { for some } u \in S a \cap S c, v \in b S \cap d S \text {. }
$$

Remark. If $S=S^{1}$ is naturally quasisemilatticed then $(S \times S) / \sigma$ is a semilattice under the partial order in Theorem 3.8 if and only if $(a, b) \sigma(c, d)$ implies $\left(a \Lambda_{l} u, b \Lambda_{r} v\right) \sigma\left(c \Lambda_{l} u, d \Lambda_{r} v\right)$ for all $u, v \in S$ where, for example $a \Lambda_{l} u$ denotes any element of $S$ such that $S(a \wedge, u)=S a \cap S u$.

4. Naturally quasisemilatticed semigroups. If $S=S^{1}$ is a naturally quasisemilatticed semigroup then it is easy to see that an inverse semigroup $T$ is separated over $S$, by a homomorphism $\theta$, if and only if $T$ is generated as an inverse semigroup and, for each $a, b \in S$,

$$
\begin{array}{ll}
a \theta a \theta^{-1} b \theta b \theta^{-1}=\left(a \wedge_{r} b\right) \theta\left(a \wedge_{r} b\right) \theta^{-1} & \text { if }\left(a \wedge_{r} b\right) S=a S \cap b S, \\
a \theta^{-1} a \theta b \theta^{-1} b \theta=\left(a \wedge_{l} b\right) \theta^{-1}\left(a \wedge_{l} b\right) \theta & \text { if } S\left(a \wedge_{l} b\right)=S a \cap S b .
\end{array}
$$

It follows that there is a universal inverse semigroup $E(S)$ which is separated over $S ; E(S)$ is the quotient of $I(S)$ under the relations

$$
\begin{aligned}
& a a^{-1} b b^{-1}=\left(a \wedge_{r} b\right)\left(a \wedge_{r} b\right)^{-1} \text { if }\left(a \wedge_{r} b\right) S=a S \cap b S, \\
& a^{-1} a b^{-1} b=\left(a \wedge_{l} b\right)^{-1}\left(a \wedge_{l} b\right) \text { if } S\left(a \wedge_{l} b\right)=S a \cap S b .
\end{aligned}
$$

In this section we shall give an explicit construction for $E(S)$, as the inverse hull of $S \rho$ under a shift representation $\rho$ of $S$, and several coordinatisations of $E(S)$.

Throughout this section and the following ones we shall suppose that a choice of representatives has been made from the generators of the principal left 
and right ideals of the naturally quasisemilatticed semigroup being considered; if $a, b \in S$ then $a \wedge, b$ will denote the representative of the principal right ideal $a S \cap b S$ and $a \wedge_{l} b$ will denote the representative of the principal left ideal $S a \cap S b$. For each $a, b \in S$ we also choose elements $a *_{r} b$ and $a *_{l} b$ in $S$ such that $a\left(a *_{r} b\right)=a \Lambda_{r} b,\left(a *_{l} b\right) b=a \Lambda_{l} b$.

Definition. Let $S=S^{1}$ be a naturally quasisemilatticed semigroup and let $\sigma$ be an equivalence on $S \times S$. Then we shall say that $\sigma$ is a semilattice congruence on $S \times S$ if $(S \times S) / \sigma$ is a semilattice under

$(a, b) \sigma \leq(c, d) \sigma \Leftrightarrow(a, b) \sigma(u, v)$ for some $u \in S a \cap S c, v \in b S \cap d S$.

Thus $\sigma$ is a semilattice congruence if and only if, for every choice function on the generators of the principal left ideals and right ideals of $S$,

$(a, b) \sigma(c, d),(u, v) \sigma(x, y)$ implies $\left(a \wedge, u, b \wedge_{r} v\right) \sigma\left(c \wedge_{l} x, d \wedge_{r} y\right)$.

Lemma 4.1. Let $S=S^{1}$ be a naturally quasisemilatticed semigroup and let $\sigma$ be a semilattice congruence on $S$ which obeys (1). Define a relation $\sigma^{*}$ on $S \times S$ by

$$
(a, b) \sigma^{*}(c, d) \Leftrightarrow(a, b) \sigma(c, d) \sigma(u, v) \text { for some } u, v \in S
$$

such that $a v=c v, u b=u d$. Then $\sigma^{*}$ is an equivalence on $S \times S$ which obeys (1) and

$$
(a, b) \sigma^{*}(c, d) \Leftrightarrow(a, b) \sigma^{*}(x, y) \text { for some } x \in S a \cap S c, y \in b S \cap d S
$$

in particular, $\sigma^{*}$ is a shift.

Proof. First of all, $\sigma^{*}$ is clearly reflexive and symmetric. Suppose that $(a, b) \sigma^{*}(c, d)$ and $(c, d) \sigma^{*}(e, f)$. Then there exist $x, y, u, v, \in S$ such that $(a, b) \sigma(c, d) \sigma(u, v)$ with $a v=c v, u b=u d$ and $(c, d) \sigma(e, f) \sigma(x, y)$ with $c y=e y, x d=x f$. Since $\sigma$ is a semilattice congruence, $(a, b) \sigma(e, f) \sigma$ $\left(u \wedge_{l} x, v \Lambda_{r} y\right)$. Further, since $v \wedge_{r} y=v\left(v *_{r} y\right), a\left(v \wedge_{r} y\right)=a v\left(v *_{r} y\right)=$ $c v\left(v{ }_{r}, y\right)=c(v \wedge, y)$ and similarly $c(v \wedge, y)=e(v \wedge, y)$; likewise $(u \wedge, x) b=$ $\left(u \Lambda_{l} x\right) f$. Hence $(a, b) \sigma^{*}(e, f)$ and so $\sigma^{*}$ is transitive.

Suppose now that $(a, x b) \sigma^{*}(c, x d)$. Then $(a, x b) \sigma(c, x d) \sigma(u, v)$ for some $u, v \in S$ such that $a v=c v, u x b=u x d$. Then, since $\sigma$ is a semilattice congruence $(a, x b) \sigma(u, x \wedge, v)=\left(u, x\left(x *_{r} v\right)\right)$ so that $(a x, b) \sigma(c x, d) \sigma\left(u x, x *_{r} v\right)$ by (1). Further,

$$
\begin{aligned}
a x(x *, v) & =a(x \wedge, v)=a x(v *, x)=c v(v *, x)=c x(x *, v) \text { and } \\
(u x) b & =u(x b)=u(x d)=(u x) d .
\end{aligned}
$$

Hence, $(a x, b) \sigma^{*}(c x, d)$. The dual holds by symmetry so we get (1).

Next suppose that $(a, b) \sigma^{*}(c, d)$. Then it is easy to see from the definition 
of $\sigma^{*}$ that there exist $e \in S a, f \in b S$ such that $(a, b) \sigma(c, d) \sigma(e, f)$ and $e b=e d$, $a f=c f$. Since $S$ is naturally quasisemilatticed and $e b=e d \in e b S \cap e d S, e b=$ $e\left(b \wedge_{r} d\right) t$ for some $t \in S$, and, similarly $a f=s\left(a \wedge_{l} c\right) f$ for some $s \in S$. Because $\left(e \wedge_{l} a\right) \mathfrak{Q} e, f R\left(f \wedge_{r} b\right)$ and, by Theorem 3.9, $r \subseteq \sigma$, the se equations imply

$$
(a, b) \sigma(e, b) \sigma\left(e,\left(b \wedge_{r} d\right) t\right) \text { and }(a, b) \sigma\left(s\left(a \wedge_{l} c\right), f\right)
$$

Set

$$
u^{\prime}=s\left(a \wedge_{l} c\right) \wedge_{l} e, \quad v^{\prime}=f \wedge_{r}\left(b \wedge_{r} d\right) t
$$

Then, since $\sigma$ is a semilattice congruence and $(a, b) \sigma(s(a \wedge, c), f) \sigma(e,(b \wedge, d) t)$,

$$
(a, b) \sigma\left(s\left(a \wedge_{l} c\right) \wedge_{l} e, f \wedge_{r}\left(b \wedge_{r} d\right)_{t}\right)=\left(u^{\prime}, v^{\prime}\right) \text {. }
$$

Further

$$
s\left(a \wedge_{l} c\right) v^{\prime}=s\left(a \wedge_{l} c\right) f\left(f *_{r}\left(b \wedge_{r} d\right) t\right)=a f\left(f *_{r}\left(b \wedge_{r} d\right)_{t}\right)=a v^{\prime}
$$

and similarly $u^{\prime}\left(b \wedge_{r} d\right) t=u^{\prime} b$.

Finally, since $\left(u^{\prime}, v^{\prime}\right) \leq(s(a \wedge, c),(b \wedge, d) t) \leq(a, b)$ in the natural quasiorder on $S \times S$ and each $\sigma$ class is convex, the fact that $(a, b) \sigma\left(u^{\prime}, v^{\prime}\right)$ implies $(a, b) \sigma\left(s\left(a \wedge_{l} c\right),\left(b \wedge_{r} d\right) t\right)$. Hence we have shown

$(a, b) \sigma\left(s\left(a \wedge_{l} c\right),\left(b \wedge_{r} d\right) t\right) \sigma\left(u^{\prime}, v^{\prime}\right)$ and $a v^{\prime}=s\left(a \wedge_{l} c\right) v^{\prime}, u^{\prime} b=u^{\prime}\left(b \wedge_{r} d\right)_{t}$; that is $(a, b) \sigma^{*}\left(s\left(a \wedge_{l} c\right),\left(b \wedge_{r} d\right) t\right)$. Thus (3) holds.

Lemma 4.2. Let $S=S^{1}$ be a naturally quasisemilatticed semigroup and let $\sigma$ be an equivalence on $S \times S$ which obeys (1) and (3). Suppose that $\rho$ is the corresponding shift representation of $S$. Then the inverse bull of $S \rho$ in $9((S \times S) / \sigma)$ is separated over $S$ by $\rho$.

Further the semilattice congruence $\sigma_{E}$ defined by

$$
(a, b) \sigma_{E}(c, d) \Leftrightarrow \rho_{a}^{-1} \rho_{a} \rho_{b} \rho_{b}^{-1}=\rho_{c}^{-1} \rho_{c} \rho_{d} \rho_{d}^{-1}
$$

is contained in every semilattice congruence which contains $\sigma$.

Proof. Let $a, b \in S$; then $\Delta \rho_{a}=\{(x, a y) \sigma: x, y \in S\}$ and so, since $\sigma$ obeys

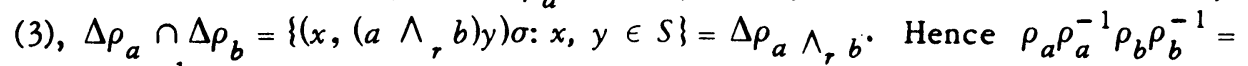
$\rho_{a} \wedge_{r} \rho_{a} \rho_{r} b$ and dually. Thus the inverse hull of $S \rho$ is separated over $S$ by $\rho$.

By Lemma 3.4, $\sigma_{E}$ is a semilattice congruence on $S \times S$. Suppose that $\pi$ is also a semilattice congruence and that $\sigma \subseteq \pi$. Then $(a, b) \sigma_{E}(c, d)$ implies $(a, b) \pi(x c, d y),(c, d) \pi(u a, b v)$ for some $x, y, u, v \in S$ 
and so, since $\pi$ is a semilattice congruence, $(a, b) \pi(c, d)$. Hence $\sigma_{E} \subseteq \pi$.

It follows from Lemma 4.2 that, if $\sigma$ is a semilattice congruence on $S \times S$ which obeys (1), then $\sigma_{E}^{*} \subseteq \sigma$. However $\sigma$ need not equal $\sigma_{E}^{*}$. (For example, if $S$ is cancellative with trivial group of units $\sigma_{E}^{*}$ is always the identity while $\sigma$ could be $S \times S$ ). However, if we take $\sigma=\tau$ then, since, by Theorem 3.8, $\tau$ is the smallest semilattice congruence which obeys $(1), \tau=\tau_{E}^{*}$. We can use this to find $E(S)$.

The next lemma is rather technical. It can be applied, among other things, to give necessary and sufficient conditions for embedding naturally quasisemilatticed semigroups in inverse semigroups.

Lemma 4.3. Let $S=S^{1}$ be a semigroup and define an equivalence $\tau$ on $S \times S$ by $(a, b) \tau(c, d)$ if and only if there exist finite sets $x_{0}, \cdots, x_{n^{n}} y_{0}, \cdots, y_{n}$ in $S$ with $a=x_{0}, c=x_{n}, b=y_{0}, d=y_{n}$ and $x_{i-1} y_{i-1} \mathfrak{Q}_{i} y_{i-1} R_{x_{i} y_{i}}, 1 \leq i \leq n$. Let $b=u a=c v, e=p d=f q$ and suppose there exist $x, y, \alpha, \beta, \gamma, \delta$ in $S$ such that

$$
(u, a) \tau(x p, d y) \tau(a, \beta) \text { with } u \beta=x p \beta, \alpha a=\alpha d y
$$

and

$$
(c, v) \tau(x f, q y) \tau(y, \delta) \text { with } c \delta=x f \delta, \gamma v=\gamma q y \text {. }
$$

Then

$$
a b^{-1} c \leq d e^{-1} f \text { in the free inverse semigroup } I(S) \text { on } S \text {. }
$$

Proof. Let $\sigma$ be defined on $S \times S$ by $(a, b) \sigma(c, d)$ if and only if

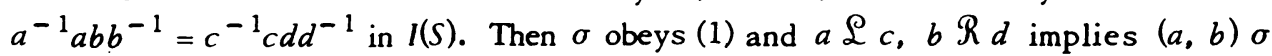
$(c, d)$. As in the proof of Theorem 3.7, this implies $\tau \subseteq \sigma$.

In $I(S)$ :

$$
\begin{aligned}
a b^{-1} c & =a a^{-1} u^{-1} c=a a^{-1} u^{-1} u u^{-1} c \\
& =d y(d y)^{-1}(x p)^{-1}(x p) \beta \beta^{-1} u^{-1} c \text { since }(u, a) \tau(x p, d y) \tau(\alpha, \beta) \\
& =d y(x p d y)^{-1} u \beta(u \beta)^{-1} c=d y(x f q y)^{-1} u \beta(u \beta)^{-1} c \\
& \leq d y(x f q y)^{-1} c \text { since } u \beta(u \beta)^{-1} \text { is idempotent. }
\end{aligned}
$$

Now, since $(x f, q y) \tau(\gamma, \delta)$ and $\tau \subseteq \sigma$,

$$
(x f)^{-1} x f q y(q y)^{-1}=y^{-1} \gamma \delta \delta^{-1}
$$

so that

$$
(x f)^{-1} x f q y(q y)^{-1}=(x f)^{-1} x f y^{-1} \gamma \delta \delta^{-1} q y(q y)^{-1}
$$

which implies $x f q y=x f \gamma^{-1} \gamma \delta \delta^{-1} q y$. Thus 


$$
\begin{aligned}
a b^{-1} c & \leq d y\left(x f \gamma^{-1} y \delta \delta^{-1} q y\right)^{-1} c=d y\left(x f \delta \delta^{-1} \gamma^{-1} \gamma q y\right)^{-1} c \\
& =d y\left(c \delta \delta^{-1} \gamma^{-1} \gamma q y\right)^{-1} c=d y y^{-1} q^{-1} \gamma^{-1} \gamma \delta \delta^{-1} c^{-1} c \\
& =d y y^{-1} q^{-1}(x f)^{-1} x f q y(q y)^{-1} \text { since }(c, v) \tau(x f, q y) \tau(y, \delta) \text { and } \tau \subseteq \sigma \\
& =d y y^{-1} q^{-1}(x f)^{-1} x f=d y y^{-1}(f q)^{-1} x^{-1} x f \\
& \leq d e^{-1} f \text { since } e=f q .
\end{aligned}
$$

Theorem 4.4. Let $S=S^{1}$ be a naturally quasisemilatticed semigroup and let $\rho: S \rightarrow g\left((S \times S) / \tau^{*}\right)$ be the shift representation of $S$ associated with $\tau^{*}$. Then the inverse bull of $S \rho$ in $g\left((S \times S) / \tau^{*}\right)$ is isomorpbic to the quotient $E(S)$ of $I(S)$ modulo the relations

$$
a a^{-1} b b^{-1}=\left(a \wedge_{r} b\right)\left(a \wedge_{r} b\right)^{-1}, \quad a^{-1} a b^{-1} b=\left(a \wedge_{l} b\right)^{-1}\left(a \wedge_{l} b\right)
$$

for all $a, b \in S$.

Proof. The proof of Lemma 4.2 shows that, for $a, b \in S$,

$$
\left.\left.\rho_{a} \rho_{a}^{-1} \rho_{b} \rho_{b}^{-1}=\rho_{(a \wedge} \wedge_{r} \rho_{(a}^{-1} \wedge_{r} b\right), \quad \rho_{a}^{-1} \rho_{a} \rho_{b}^{-1} \rho_{b}=\rho_{\left(a \wedge_{l} b\right)}^{-1} \rho_{(a \wedge} b\right)
$$

so that the inverse hull $T$ of $S \rho$ is a quotient of $E(S)$. More precisely, there is a unique homomorphism $\psi: E(S) \rightarrow T$ such that $\rho=\mu \psi$ where $\mu$ denotes the canonical homomorphism $S \rightarrow E(S)$.

Let $b=u a=c v, e=p d=f q$ and suppose that $\rho_{a} \rho_{b}^{-1} \rho_{c} \leq \rho_{d} \rho_{e}^{-1} \rho_{f}$. Then since, for example, $\Delta \rho_{a} \rho_{b}^{-1} \rho_{c}=\left\{(x u, a y) \tau^{*}: x, y \in S\right\}$, there exist $x, y \in S$ such that $(u, a) \tau^{*}(x p, d y)$ and $(u, a) \tau^{*} \rho_{a} \rho_{b}^{-1} \rho_{c}=(x p, d y) \tau^{*} \rho_{d} \rho_{e}^{-1} \rho_{f}$; that is $(c, v) \tau^{*}(x f, q y)$. The first and third of these relations are precisely those in Lemma 4.3. Hence, in $I(S), a b^{-1} c \leq d e^{-1} \%$. Since $E(S)$ is a quotient of $I(S)$, we have there $a \mu b \mu^{-1} c \mu \leq d \mu e \mu^{-1} f \mu$. Therefore $\left(a \mu b \mu^{-1} c \mu\right) \psi=\left(d \mu e \mu^{-1} d \mu\right) \psi$ implies $a \mu b \mu^{-1} c \mu=d \mu e \mu^{-1} f \mu$ and so $\psi$ is one-to-one; thus an isomorphism.

If $S=S^{1}$ is a semigroup whose principal left and right ideals form chains then the relations

$$
a a^{-1} b b^{-1}=\left(a \wedge_{r} b\right)\left(a \wedge_{r} b\right)^{-1}, \quad a^{-1} a b^{-1} b=\left(a \wedge_{l} b\right)^{-1}\left(a \wedge_{l} b\right)
$$

hold in $I(S)$. Hence we have

Theorem 4.5. Let $S=S^{1}$ be a semigroup whose principal left and right ideals form chains under inclusion and let $\rho$ be the shift representation of $S$ associated with $\tau^{*}$. Then $I(S)$ is isomorphic to the inverse bull of $S \rho$ in $g\left((S \times S) / \tau^{*}\right)$.

As a consequence of its description as a subsemigroup of $9\left((S \times S) / \tau^{*}\right)$, the semigroup $E(S)$ admits several natural coordinatisations. Before giving these, 
we show how $E(S)$ can be used to give necessary and sufficient conditions for embedding a naturally quasisemilatticed semigroup in an inverse semigroup.

Theorem 4.6. Let $S=S^{1}$ be a naturally quasisemilatticed semigroup. Then $S$ can be embedded in an inverse semigroup if and only if the canonical homomor. phism $\mu: S \rightarrow E(S)$ is one-to-one.

Proof. Let $\eta$ be the canonical homomorphism $S \rightarrow I(S)$. Then, since $\mu$ can be factored through $\eta, \eta \circ \eta^{-1} \subseteq \mu \circ \mu^{-1}$. On the other hand, $a \mu=b \mu$ implies $a \mu a \mu^{-1} a \mu=b \mu b \mu^{-1} b \mu$ in $E(S)$ and so, by Lemma 4.2, $a a^{-1} a=b b^{-1} b$ in $I(S)$. Thus $a \mu=b \mu$ implies $a \eta=b \eta$. Hence $\eta \circ \eta^{-1}=\mu \circ \mu^{-1}$.

Theorem 4,7. Let $S=S^{1}$ be a naturally quasisemilatticed semigroup and let $U$ be the set of all 4-tuples $(a, v, u, c)$ of elements of $S$ with ua $=c v$. Define a binary operation on $U$ by

$$
(a, v, u, c)(d, q, p, f)=\left(a\left(v *{ }_{r} d\right), q\left(d *{ }_{r} v\right),\left(p *{ }_{l} c\right) u,\left(c *_{l} p\right) f\right) .
$$

Furtber define

$$
(a, v, u, c) \sim(d, q, p, f) \Leftrightarrow \text { there exist } x, y, z, w \in S
$$

sucb that $(u, a) \tau^{*}(x p, d y),(c, v) \tau^{*}(x f, q y),(p, d) \tau^{*}(z u, a w),(f, q) \tau^{*}(z c, v w)$.

Then $\sim$ is a congruence on $U$ and $U / \sim$ is isomorphic to $E(S)$.

Proof. First of all, it is easy to see that the multiplication described above is, in fact, a binary operation on $U$. Define $\psi: U \rightarrow E(S)$ by $(a, v, u, c) \psi=$ $\rho_{a} \rho_{b}^{-1} \rho_{c}$ where $b=u a=c v$; since $E(S)$ is, by Theorem 3.2, an inverse semigroup of strong quotients of $S \rho, \psi$ is onto. Further, easy calculation shows that $\Delta \rho_{a} \rho_{b}^{-1} \rho_{c}=\left\{(x u, a y) \tau^{*}: x, y \in S\right\}, \nabla \rho_{a} \rho_{b}^{-1} \rho_{c}=\left\{(x c, v y) \tau^{*}: x, y \in S\right\}$ and thus, because $\tau^{*}$ obeys (3), that

$$
\begin{aligned}
& \Delta \rho_{a} \rho_{b}^{-1} \rho_{c} \rho_{d} \rho_{e}^{-1} \rho_{f}=\left\{\left(x\left(p *{ }_{l} c\right) u, a\left(v *{ }_{r} d\right) y\right) \tau^{*}: x, y \in S\right\}, \\
& \nabla \rho_{a} \rho_{b}^{-1} \rho_{c} \rho_{d} \rho_{e}^{-1} \rho_{f}=\left\{\left(x\left(c *{ }_{l} p\right) f, q(d *, v) y\right) \tau^{*}: x, y \in S\right\} .
\end{aligned}
$$

Thus, because of the action of $\rho_{a} \rho_{b}^{-1} \rho_{c} \rho_{d} \rho_{e}^{-1} \rho_{f}$ we find

$$
\begin{aligned}
\rho_{a} \rho_{b}^{-1} \rho_{c} \rho_{d} \rho_{e}^{-1} \rho_{f} & =\rho_{\left(p{ }_{l}, c\right) u} \rho_{\left(p *_{l} c\right) u a\left(v{ }_{r} d\right)}^{1} \rho_{\left(c *_{l} \rho\right) f} \\
& =[(a, v, u, c)(d, q, p, f)] \psi .
\end{aligned}
$$

Hence $\psi$ is a homomorphism.

Finally, the proof of Theorem 4.4 shows that $\rho_{a} \rho_{b}^{-1} \rho_{c}=\rho_{d} \rho_{e}^{-1} \rho_{f}$ if and only if $(a, v, u, c) \sim(d, q, p, f)$. Hence $\sim$ is the congruence of $\psi$ and so $U / \sim$ is isomorphic to $E(S)$. 
Theorem 4.8. Let $S=S^{1}$ be a naturally quasisemilatticed semigroup and let $V$ be the set of all triples $(a, b, c)$ of elements of $S$ witb $b \in S a \cap c S$. Define a binary operation on $V$ by

$$
(a, b, c)(d, e, f)=\left(a(b *, c d),\left(e *_{l} c d\right) c d(c d *, b),(c d *, e) f\right)
$$

and a relation $\sim$ on $V$ by

$$
(a, b, c) \sim(d, e, f) \hookrightarrow b=u a=c v, e=p d=f q \text { and there exist } x, y, z, w \in S
$$

sucb that $(u, a) \tau^{*}(x p, d y),(c, v) \tau^{*}(x f, q y),(p, d) \tau^{*}(z u, a w),(f, q) \tau^{*}(z c, v w)$.

Then $\sim$ is a congruence on $V$ and $V / \sim$ is isomorphic to $E(S)$.

\section{Proof. First}

$$
\left(e *{ }_{l} c d\right) c d\left(c d *{ }_{r} b\right)=\left(e *{ }_{l} c d\right) b\left(b *{ }_{r} c d\right)=\left(e *{ }_{l} c d\right) u a\left(b *{ }_{r} c d\right) \in S a\left(b *{ }_{r} c d\right)
$$

while

$$
\left(e *{ }_{l} c d\right) c d(c d *, b)=\left(c d *{ }_{l} e\right) e\left(c d *{ }_{r} b\right)=\left(c d *_{l} e\right) f q\left(c d *_{r} b\right) \in\left(c d *_{l} e\right) f s
$$

so that the multiplication is a binary operation on $V$.

Define $\psi: E(S)$ by $(a, b, c) \psi=\rho_{a} \rho_{b}^{-1} \rho_{c}$. Then, by Theorem $3.2, \psi$ is onto and further, from the proof of that theorem, $\psi$ is a homomorphism. Finally, as in the proof of Theorem 4.7, $\sim$ is the congruence of $\psi$ so that $E(S) \approx V / \sim$.

The coordinatisation given in Theorem 4.8 reduces to that given by Eberhart and Selden when $S$ is a subsemigroup of the positive reals $\leq 1$ [5]. It has, however, the drawback that, when restricted to a Brandt $\mathcal{G}$-class of $E(S)$ it does not give the usual Brandt multiplication. The latter can be recovered if we give $E(S)$ the coordinates described in the next theorem.

Theorem 4.9. Let $S=S^{1}$ be a naturally quasisemilatticed semigroup and let $W$ be the set of all triples $(a, b, c)$ of elements of $S$ with $b \in S a \cap S c$. Define $a$ binary operation on $W$ by

$$
(a, b, c)(d, e, f)=\left(a\left(c *_{r} d\right), b\left(c *_{r} d\right) \wedge_{l} e\left(d *_{r} c\right), f\left(d *{ }_{r} c\right)\right)
$$

and a relation $\sim$ by

$$
(a, b, c) \sim\left(d, e_{,} f\right) \Leftrightarrow b=u a=v c, e=p d=q f \text { and there exist } x, y, z, w \in S
$$

sucb that $(u, a) \tau^{*}(x p, d y),(v, c) \tau^{*}(x q, f y),(p, d) \tau^{*}(z u, a w),(q f) \tau^{*}(z v, c w)$.

Then $\sim$ is a congruence on $W$ and $E(S) \approx W / \sim$.

Proof. Since

$$
\begin{aligned}
b\left(c *_{r} d\right) \wedge_{l} e\left(d *{ }_{r} c\right) & =\left\{b\left(c *_{r} d\right) *{ }_{l} e\left(d *_{r} c\right)\right\} q f\left(d *_{r} c\right) \\
& =\left\{e\left(d *{ }_{r} c\right) *{ }_{l} b\left(c *{ }_{r} d\right)\right\} u a(c *, d)
\end{aligned}
$$


where $b=u a=v c, e=p d=q f$, the multiplication described is, in fact, a binary operation on $W$.

Define $(a, b, c) \psi=a \rho(b \rho)^{-1} v \rho$ if $b=v c$. Then, firstly, $\psi$ is well defined. For, if $b=v c=w c$, then

$$
\begin{aligned}
a \rho(b \rho)^{-1} v \rho & =a \rho(v \rho c \rho)^{-1} v \rho=a \rho c \rho^{-1} v \rho^{-1} v \rho \\
& =a \rho c \rho^{-1} v \rho^{-1} v \rho c \rho c \rho^{-1} \quad \text { since indempotents commute } \\
& =a \rho(v c) \rho^{-1}(v c) \rho c \rho^{-1}=a \rho(w c) \rho^{-1}(w c) \rho c \rho^{-1}=a \rho(b \rho)^{-1} w \rho .
\end{aligned}
$$

Next we show that $\psi$ is a homomorphism of $W$ onto $E(S)$; the ontoness is obvious.

Since $(a, b, c) \psi(d, e, f) \psi=\left(a \rho(b \rho)^{-1} v \rho\right)\left(d \rho(e \rho)^{-1} q \rho\right)$, it follows from the multiplication in $\mathrm{g}\left((S \times S) / \tau^{*}\right)$ that

$$
(a, b, c) \psi(d, e, f) \psi=\left\{a\left(c *_{r} d\right)\right\} \rho\left\{\left(\rho \wedge_{l} v\right)\left(d \wedge_{r} c\right)\right\} \rho^{-1}\left\{\left(v *_{l} \rho\right) q\right\} \rho .
$$

On the other hand, from the multiplication in $W$.

$\{(a, b, c)(d, e, f)\} \psi$

$$
=\left\{a\left(c *{ }_{r} d\right)\right\} \rho\left\{b\left(c *_{r} d\right) \wedge_{l} e\left(d *_{r} c\right)\right\} \rho^{-1}\left(\left\{b\left(c *_{r} d\right) *{ }_{l} e\left(d *_{r} q\right)\right\} q\right) \rho .
$$

Since $S$ is naturally quasisemilatticed,

$$
\left(p \wedge_{l} v\right)\left(d \Lambda_{r} c\right) \mathfrak{L}\left\{p\left(d \wedge_{r} c\right) \wedge_{l} v\left(d \Lambda_{r} c\right)\right\}=e\left(d *_{r} c\right) \wedge_{l} b\left(c *_{r} d\right)
$$

so there exist $x, z \in S$ such that

$$
\begin{aligned}
\left(p \wedge_{l} v\right)\left(d \wedge_{r} c\right) & =x\left\{b\left(c *{ }_{r} d\right) \wedge_{l} e\left(d *_{r} c\right)\right\} \\
z\left\{\left(p \wedge_{l} v\right)\left(d \wedge_{r} c\right)\right\} & =b\left(c *_{r} d\right) \wedge_{l} e\left(d *_{r} c\right) .
\end{aligned}
$$

Hence, working with $x$ alone,

$$
\left(\left(p \wedge_{l} v\right)\left(d \wedge_{r} c\right), 1\right) \tau^{*}\left(x\left\{b\left(c *_{r} d\right) \wedge_{l} e\left(d *_{r} c\right)\right\}, 1\right)
$$

so that, since $\tau^{*}$ is a shift and

$$
\begin{aligned}
(p \wedge, v)(d \wedge, c)=(p * l v) u a(c *, d)=(v *, p) q f\left(d *_{r} c\right) \\
b\left(c *_{r} d\right) \wedge_{l} e(d *, c)=\left\{e\left(d *_{r} c\right) * l b\left(c *_{r} d\right)\right\} u a\left(c *_{r} d\right) \\
=\left\{b(c *, d) * *_{l} e\left(d *_{r} c\right)\right\} q f\left(d *_{r} c\right)
\end{aligned}
$$

we get

$$
\begin{aligned}
& \left((p *, v) u, a\left(c *{ }_{r} d\right)\right) \tau^{*}\left(x\left\{e\left(d *_{r} c\right) *, b\left(c *_{r} d\right)\right\} u, a\left(c *_{r} d\right)\right\}, \\
& \left(\left(v * *_{l} p\right) q, f\left(d * *_{r} c\right)\right) \tau^{*}\left(x\left\{b\left(c *_{r} d\right) *, e\left(d * *_{r} c\right)\right\} q, f\left(d *_{r} c\right)\right\} .
\end{aligned}
$$

Hence, by Lemma 4.3,

$$
(a, b, c) \psi(d, e, f) \psi \leq[(a, b, c)(d, e, f)] \psi .
$$


Operating with $z$ gives the reverse inqeuality so that $\psi$ is a homomorphism.

Finally, if $b=u a=v c, e=p d=q f$, Lemma 4.3 and the definition of $\rho$ shows that

$$
(a, b, c) \psi=(d, e, f) \psi \Leftrightarrow(a, b, c) \sim(d, e, f) .
$$

Hence $E(S) \approx W / \sim$.

The congruences in Theorems 4.7, 4.8, 4.9, and thus the coordinatisations for $E(S)$, undergo considerable simplification in two cases: (i) $S$ is cancellative; the results for this case are stated in Theorem 6.2. (ii) $\mathcal{T}$ is trivial on $S$; in this case $\tau=\tau^{*}=\tau_{0}$ is a semilattice congruence on $S \times S$ and the congruences reduce to

$$
\begin{aligned}
& (a, v, u, c) \sim(d, q, p, f) \text { in } U \subseteq(u, a) \tau_{0}(p, d),(c, v) \tau_{0}(f, q) \text {, } \\
& (a, b, c) \sim(d, e, f) \text { in } V \Leftrightarrow(u, a) \tau_{0}(p, d),(c, i) \tau_{0}(f, q) \\
& \text { where } b=u a=c v, e=p d=f q, \\
& (a, b, c) \sim(d, e, f) \text { in } W \Leftrightarrow(u, a) \tau_{0}(p, d),(v, c) \tau_{0}(q, f) \\
& \text { where } b=u a=v c, c=p d=q f \text {. }
\end{aligned}
$$

To end this section, we give an example to show how the coordinatisation in Theorem 4.9 gives rise to the Brandt multiplication in Brandt $\mathscr{Y}$-classes of $E(S)$. Suppose that $S \times S^{1}$ is a naturally quasisemilatticed cancellative semigroup on which $\mathcal{G}$ is trivial. Then it follows from Theorem 5.2 that, in $E(S)=W / \sim$,

$$
J_{b}=\left\{(a, b, c): b \in S a \cap S_{c}\right\}
$$

is a $\mathscr{G}$-class for each $b \in S:$ in this case $\sim$ is, in fact, the identity congruence. By Theorem 4.9,

$$
(a, b, c)(d, b, f)=\left(a\left(c *{ }_{r} d\right), b\left(c *{ }_{r} d\right) \wedge, b\left(d *{ }_{r} c\right), f\left(d *{ }_{r} c\right)\right) .
$$

This belongs to $J_{b}$ if and only if $b=b\left(c *_{r} d\right) \wedge_{l} b\left(d *_{r} c\right)$. But the latter implies $b \in S b\left(c *_{r} d\right) S \subseteq S b S$ and $b \in S b\left(d *_{r} c\right) S \subseteq S b S$ whence, since $\mathcal{G}$ is trivial and $S$ is cancellative, $\left(c{\underset{r}{*}}_{d}\right)=1=\left(d{ }_{r}^{*} c\right)$; thus $c=d$. Hence, modulo the ideal generated by $J_{b}$,

$$
(a, b, c)(d, \bar{b}, f)= \begin{cases}(a, b, f) & \text { if } c=d, \\ 0 & \text { otherwise. }\end{cases}
$$

This is just the multiplication in the Brandt semigroup

$$
\mathbb{M}^{0}(\{1\} ; X, X, \Delta) \text { where } X=\{x \in S: b \in S x\} \text {. }
$$

5. Green's relations and congruences on $E(S)$. In this section $S=S^{1}$ denotes a naturally quasisemilatticed semigroup and $E(S)$ denotes the quotient of $I(S)$, modulo the relations

$$
a a^{-1} b b^{-1}=\left(a \wedge_{r} b\right)\left(a \wedge_{r} b\right)^{-1}, \quad a^{-1} a b^{-1} b=\left(a \wedge_{l} b\right)^{-1}\left(a \wedge_{l} b\right)
$$


for all $a, b \in S$, regarded as a subsemigroup of $9\left((S \times S) / \tau^{*}\right)$. The results are easily translated into the coordinatised forms of $E(S)$.

Lemma 5.1. Let $\rho_{a} \rho_{b}^{-1} \rho_{c} \in E(S)$ where $b=u a=c v$. Then

(i) $\left(\rho_{a} \rho_{b}^{-1} \rho_{c}\right)^{-1}\left(\rho_{a} \rho_{b}^{-1} \rho_{c}\right)=\rho_{c}^{-1} \rho_{c} \rho_{v} \rho_{v}^{-1}$,

(ii) $\left(\rho_{a} \rho_{b}^{-1} \rho_{c}\right)\left(\rho_{a} \rho_{b}^{-1} \rho_{c}\right)^{-1}=\rho_{u}^{-1} \rho_{u} \rho_{a} \rho_{a}^{-1}$.

Theorem 5.2. Let $\rho_{a} \rho_{b}^{-1} \rho_{c}, \rho_{d} \rho_{e}^{-1} \rho_{f} \in E(S)$ where $b=u a=c v, e=p d \doteq / q$.

(i) $\rho_{a} \rho_{b}^{-1} \rho_{c} \mathcal{L} \rho_{d} \rho_{e}^{-1} \rho_{f} \Leftrightarrow(c, v) \tau(f, q)$.

(ii) $\rho_{a} \rho_{b}^{-1} \rho_{c} R \rho_{d} \rho_{e}^{-1} \rho_{f} \Leftrightarrow(u, a) \tau(p, d)$.

(iii) $\rho_{a} \rho_{b}^{-1} \rho_{c} \mathcal{H} \rho_{d} \rho_{e}^{-1} \rho_{f} \Leftrightarrow(u, a) \tau(p, d),(c, v) \tau(f, q)$.

(iv) $\rho_{a} \rho_{b}^{-1} \rho_{c} \mathfrak{I} \rho_{d} \rho_{e}^{-1} \rho_{f} \Leftrightarrow b \mathfrak{I} e$.

(v) $\rho_{a} \rho_{b}^{-1} \rho_{c} \leq{ }_{g} \rho_{d} \rho_{e}^{-1} \rho_{f} \Leftrightarrow b \leq_{g} e$.

Proof. (i)

$$
\begin{aligned}
\rho_{a} \rho_{b}^{-1} \rho_{c} L \rho_{d} \rho_{e}^{-1} \rho_{f} & \Leftrightarrow\left(\rho_{a} \rho_{b}^{-1} \rho_{c}\right)^{-1}\left(\rho_{a} \rho_{b}^{-1} \rho_{c}\right)=\left(\rho_{d} \rho_{e}^{-1} \rho_{f}\right)^{-1}\left(\rho_{d} \rho_{e}^{-1} \rho_{f}\right) \\
& \Leftrightarrow \rho_{c}^{-1} \rho_{c} \rho_{v} \rho_{v}^{-1}=\rho_{f}^{-1} \rho_{f} \rho_{q} \rho_{q}^{-1} \Leftrightarrow(c, v) \tau(f, q)
\end{aligned}
$$

since, by Theorem 3.8 and Lemma 3.4, $(S \times S) / \tau$ is the semilattice of idempotents of $E(S)$.

(ii) is dual to (i) while (iii) is immediate from (i) and (ii).

(iv) If $\rho_{a} \rho_{b}^{-1} \rho_{c} \mathcal{T} \rho_{d} \rho_{e}^{-1} \rho_{f}$ then $\rho_{a} \rho_{b}^{-1} \rho_{c} \mathcal{L} \rho_{x} \rho_{y}^{-1} \rho_{z} R \rho_{d} \rho_{e}^{-1} \rho_{f}$ for some $x, y, z \in S$ with $y=r x=z s$. By (i) and (ii), these imply $(c, v) \tau(z, s),(r, x) \tau$ $(p, d)$. Hence, from the definition of $r, b=c v \mathfrak{I} z s=r x \mathfrak{I} p d=e$.

Conversely, if $b \mathfrak{L} e$ then, for some $t \in S, b \mathcal{L} t R e$. Hence there exist $\alpha, \beta, \gamma, \delta \in S$ such that

$$
b=\alpha t, t=\beta b=e \gamma, e=t \delta
$$

thus $e=\beta b \delta$. Let $g=\beta u, x=a \delta$ and set $y=g x, z=f$; so $y=e=z q$. Then $u a=b \mathscr{L} t=\beta u a=g a, t \mathfrak{R} e=t \delta=\beta u a \delta=g x$. That is, ua $\mathfrak{L} a \mathfrak{R} g x$ which implies $(u, a) \tau(g, x)$. Hence, by (i), (ii),

$$
\rho_{a} \rho_{b}^{-1} \rho_{c} R \rho_{x} \rho_{y}^{-1} \rho_{f} \rho_{f}^{-1} \rho_{f} \rho_{q} \rho_{q}^{-1} \rho_{\rho_{d}} \rho_{e}^{-1} \rho_{f} \text {. }
$$

Thus $\rho_{a} \rho_{b}^{-1} \rho_{c} \mathscr{D} \rho_{d} \rho_{e}^{-1} \rho_{f}$.

If $\rho_{a} \rho_{b}^{-1} \rho_{c} \in E(S) \rho_{d} \rho_{e}^{-1} \rho_{f} E(S)$ then $\rho_{a} \rho_{b}^{-1} \rho_{c} R \rho_{x} \rho_{y}^{-1} \rho_{z}$ and $\rho_{x} \rho_{y}^{-1} \rho_{z} \epsilon$ $E(S) \rho_{d} \rho_{e}^{-1} \rho_{f}$ for some $x, y, z \in S$ with $y=r x=z s$. Since $(S \times S) / \tau$ is the semilattice of idempotents $E(S)$, these relations imply $(u, a) \tau(r, x)$ and $(z, s) \tau\left(z \wedge_{l} f, s \wedge_{r} q\right)$. Hence $b=u a \mathfrak{L} r x=y$ and $y=z s \mathscr{L}\left(z \wedge_{l} f\right)\left(s \wedge_{r} q\right)=$ $\left(z *_{l} f\right) f q\left(q *_{r} s\right)$ which implies $b \in S f q S=S e S$. 
Conversely, if $b \in$ SeS, $\rho_{b} \in E(S) \rho_{e} E(S)$ and so, since $\rho_{a} \rho_{b}^{-1} \rho_{c} \mathcal{I} \rho_{b}$ and $\rho_{d} \rho_{e}^{-1} \rho_{f} \mathcal{T} \rho_{e}, \rho_{a} \rho_{b}^{-1} \rho_{c} \leq{ }_{g} \rho_{d} \rho_{e}^{-1} \rho_{f}$.

Corollary 5.3. Let $I$ be an ideal of $S$ and set $I^{*}=\left\{\rho_{a} \rho_{b}^{-1} \rho_{c} \in E(S): b \in I\right\}$. Then $I^{*}$ is an ideal of $E(S)$ and each ideal of $E(S)$ bas this form.

Corollary 5.4. If $S$ has a kernel, so bas $E(S)$; the kernel of $E(S)$ is bisimple if the kernel of $S$ is a $\mathcal{T}$-class of $S$ (even if $\operatorname{Ker} S$ is not bisimple).

An equivalence relation $\beta$ on the set $E$ of idempotents of an inverse semigroup $T$ is called a normal partition if there is a congruence $\rho$ on $T$ such that $\beta=\rho \cap(E \times E)$. Reilly and Scheiblich [14] have shown that an equivalence $\beta$ on $E$ is a normal partition if and only if

(i) $(a, b) \in \beta,(c, d) \in \beta$ implies $(a \wedge c, b \wedge d) \in \beta$,

(ii) $(a, b) \in \beta$ implies $\left(x^{-1} a x, x^{-1} b x\right) \in \beta$ for all $a, b, c, d \in E, x \in S$.

It is shown in [14] that the mapping $\Theta: \sigma \rightarrow \sigma \cap(E \times E)$ is a complete lattice homomorphism of the complete lattice $\Lambda$ of congruences on $T$ onto the complete lattice of normal partitions on $E$. Thus each $\Theta$-class is a complete sublattice of $\Lambda$; in particular, it has a greatest and a least element; if $\beta$ is a normal partition on $E$ we shall denote the greatest and least elements of $\beta \Theta^{-1}$ by $\beta^{V}$ and $\beta^{\wedge}$ respectively.

Theorem 5.5. The lattice of $\Theta$-classes of congruences of $E(S)$ is isomorpbic to the lattice of semilattice congruences on $S \times S$ which obey (1).

If $\beta$ is the normal partition corresponding to the semilattice congruence $\sigma$ on $S \times S$ then $E(S) / \beta^{V}$ is isomorphic to the inverse bull of $S \rho$ in $g((S \times S) / \sigma)$, where $\rho$ is the shift representation of $S$ associated with $\sigma$.

Proof. Since every homomorphic image of $E(S)$ is separated over $S$, it is immediate from Theorem 3.6 and Lemma 3.4 that the normal partitions on $E(S)$ are precisely the shift semilattice congruences on $S \times S$. Further, from its definition, $E(S) / \beta^{\vee}$ is, up to isomorphism, the only fundamental homomorphic image of $E(S)$ with normal partition $\beta$. Hence the rest of the theorem follows from Theorem 3.6.

As a consequence of Theorem 5.5, we can regard the normal partitions $\beta$ of $E(S)$, and the corresponding semigroups $E(S) / \beta^{\bigvee}$, as known. Although Theorem 3.8 gives a method for constructing all shift semilattice congruences on $S \times S$ from equivalences on $S$, it does not give a unique method of construction. Hence the situation is not entirely satisfactory. However, in the case when $S$ is the positive cone of an archimedean ordered group, it is easy to see that congruences on $S$ which obey the conditions of Theorem 3.8 are the Rees factor congruences on $S$. This, together with the fact that a semigroup, with a left and right zero, has a zero, gives Theorem 4.4 of [5]. 
6. The cancellative case. If the semigroup $S \times S^{1}$ is cancellative, the theory in the previous two sections undergoes considerable simplification.

Lemma 6.1. Let $S=S^{1}$ be a cancellative naturally quasisemilatticed semigroup. Then $(a, b) \tau(c, d) \Leftrightarrow a=g c, b=d b$ for some units $g, b \in S$ wbile $\tau^{*}$ is the identity on $S \times S$.

Hence the results in Theorems 4.7, 4.8, 4.9 reduce to the results in Theorem 6.2.

Theorem 6.2. Let $S=S^{1}$ be a cancellative naturally quas isemilatticed semigroup.

(i) Let $U=\{(a, v, u, c) \in S \times S \times S \times S: u a=c v\}$; define

$$
(a, v, u, c)(d, q, p, f)=(a(v *, d), q(d *, v),(p *, c) u,(c *, p) f)
$$

and

$$
\begin{aligned}
(a, v, u, c) \sim(d, q, p, f) \Leftrightarrow u=g p, c=g f, a=d b, v=q b \\
\\
\quad \text { for some units } g, b \in S .
\end{aligned}
$$

Then $\sim$ is a congruence on $U$ and $E(S) \approx U / \sim$.

(ii) Let $V=\{(a, b, c) \in S \times S \times S: b \in S a \cap c S\}$; define

$$
(a, b, c)(d, e, f)=\left(a\left(b *{ }_{r} c d\right),\left(e *{ }_{l} c d\right) c d\left(c d *{ }_{r} b\right),\left(c d *{ }_{l} e\right) f\right)
$$

and

$$
(a, b, c) \sim(d, e, f) \Leftrightarrow a=d b, b=g e b, c=g f \text { for some units } g, b \in S .
$$

Then $\sim$ is a congruence on $V$ and $E(S) \approx V / \sim$.

(iii) Let $W=\{(a, b, c) \in S \times S \times S: b \in S a \cap S c\}$; define

$$
(a, b, c)(d, e, f)=\left(a\left(c *{ }_{r} d\right), b\left(c *{ }_{r} d\right) \wedge_{l} e\left(d *_{r} c\right), f\left(d *{ }_{r} c\right)\right)
$$

and

$$
(a, b, c) \sim(d, e, f) \Leftrightarrow a=d b, b=g e b, c=f b \quad \text { for some units } g, b \in S .
$$

Then $\sim$ is a congruence on $W$ and $E(S) \approx W / \sim$.

Definition. An inverse semigroup $T$ is an inverse semigroup of quotients of a subsemigroup $S=S^{1}$ if each element of $T$ is of the form $a b^{-1} c$ with $a, b, c \in S$.

If $S=S^{1}$ is a cancellative semigroup in which the sets of principal left and right ideals form chains under inclusion then it follows from Theorem 4.5 that $I(S)$ is a semigroup of quotients of $S$. In fact the converse is also true. To prove this, we consider a type of representation which generalises the shift representation considered earlier.

A subset $H$ of a semigroup $S=S^{1}$ is called right consistent if $a b \in H$ 
implies $a \in H$. Suppose that $H$ is a right consistent subset of a cancellative semigroup $S=S^{1}$ and for each $a \in S$, define

$$
x \rho_{a}=x a \text { for each } x \in H \text { such that } x a \in H \text {. }
$$

Then the proof of the following lemma is straightforward.

Lemma 6.3. Let $S=S^{1}$ be a cancellative semigroup and let $H$ be a right consistent subset of $S$. Then the mapping $\rho: a \rightarrow \rho_{a}$ is a representation of $S$ by one-to-one partial transformations of $H$.

Lemma 6.4. Let $S=S^{1}$ be a cancellative semigroup and let $\omega$ be the shift representation $S$ defined by $(x, a y) \omega_{a}=(x a, y)$ for all $x, y \in S$. Then $\Delta \omega_{a}^{-1} \omega_{a} \omega_{b} \omega_{b}^{-1}=S a \times b S$.

Theorem 6.5. Let $S=S^{1}$ be a cancellative semigroup. Then the following statements are equivalent.

(i) $I(S)$ is an inverse semigroup of strong quotients of $S$.

(ii) $I(S)$ is an inverse semigroup of quotients of $S$.

(iii) The sets of principal left and right ideals of $S$ form chains under inclusion.

(iv) $S$ is naturally quasisemilaticed and $I(S)$ is naturally isomorpbic to $E(S)$.

(v) $S$ is naturally quasisemilatticed and $I(S)$ is separated over $S$.

(vi) for each $a, b \in S$ there exist $x, y \in S$ such that

$$
a a^{-1} b b^{-1}=x x^{-1}, \quad a^{-1} a b^{-1} b=y^{-1} y
$$

in $I(S)$.

Proof. Clearly (i) implies (ii) and (iii) implies (iv) implies (v) implies (vi) so we need only show that (ii) implies (iii) and (vi) implies (i).

(ii) $\Rightarrow$ (iii). Let $a, b \in S$ and set $H=\left\{x \in S: a^{2} \in x S\right.$ or $\left.a b \in x S\right\}$. Then $H$ is easily seen to be right consistent; let $\rho$ be the corresponding representation of $S$. Then $a \in \Delta \rho_{a} \rho_{a}^{-1} \cap \Delta \rho_{b} \rho_{b}^{-1}$ so that $\rho_{a} \rho_{a}^{-1} \rho_{b} \rho_{b}^{-1}$ is nonzero. By hypothesis, $\rho_{a} \rho_{a}^{-1} \rho_{b} \rho_{b}^{-1}=\rho_{x} \rho_{y}^{-1} \rho_{z}$ for some $x, y, z \in S$. Thus $a \in \rho_{a} \rho_{a}^{-1} \rho_{b} \rho_{b}^{-1}$ implies $a x=u y$ for some $u \in H$ and so $a \rho_{x} \rho_{y}^{-1} \rho_{z}=u z$. Since $\rho_{a} \rho_{a}^{-1} \rho_{b} \rho_{b}^{-1}$ is idempotent, $a=u z$ and so $u y=a x=u z x$ whence, because $S$ is cancellative, $y=z x$.

Now let $\omega$ be the represenataion of $S$ by one-to-one partial transformations of $S \times S$ given in Lemma 6.4. Since, in $I(S), a a^{-1} b b^{-1}=x x^{-1} z^{-1} z$, we have

$$
S \times(a S \cap b S)=\Delta \omega_{a} \omega_{a}^{-1} \omega_{b} \omega_{b}^{-1}=\Delta \omega_{z}^{-1} \omega_{z} \omega_{x} \omega_{x}^{-1}=S z \times x S .
$$

Thus $z$ is a unit in $S$ and so, in $I(S), z^{-1} z=1$. It follows that $\rho_{a} \rho_{a}^{-1} \rho_{b} \rho_{b}^{-1}=$ $\rho_{x} \rho_{x}^{-1}$ and so $a \in \Delta \rho_{x}$; this implies $a^{2} \in a x S$ or $a b \in a x S$. Hence $a \in x S=$ $a S \cap b S$ or $b \in x S=a S \cap b S$; that is $a S \subseteq b S$ or $b S \subseteq a S$. This shows that the 
set of principal right ideals of $S$ is a chain under inclusion. Dual arguments show that the same is true for principal left ideals so (iii) is proven.

(vi) $\Rightarrow$ (i). Suppose $a a^{-1} b b^{-1}=c c^{-1}$ in $I(S)$; then $\omega_{a} \omega_{a}^{-1} \omega_{b} \omega_{b}^{-1}=\omega_{c} \omega_{c}^{-1}$ and so, by Lemma 6.4, $a S \cap b S=c S$. Hence the set of principal right ideals of $S$ is a semilattice under inclusion and, in $I(S), a a^{-1} b b^{-1}=\left(a \wedge_{r} b\right)\left(a \wedge_{r} b\right)^{-1}$. The dual clearly holds, so we may invoke Theorem 3.2 to conclude that $I(S)$ is an inverse semigroup of strong quotients of $S$.

Theorem 6.5 can be applied to characterise the positive cones of right ordered groups among semigroups.

Theorem 6.6. Let $S=S^{1}$ be a semigroup. Then the following are equivalent.

(i) $S$ is positive cone of a right ordered group.

(ii) each element of $I(S)$ bas the form $x y^{-1} z$ for a unique triple $x, y, z \in S$ with $y \in S x: \cap z S$.

Proof. (i) $\Rightarrow$ (ii). Since $S$ is cancellative and the sets of principal left and right ideals of $S$ are chains under inclusion, it follows from Theorem 6.5 that each element of $I(S)$ has the form $x y^{-1} z$ where $y \in S x \cap z S$. Further, by Theorem 6.2, $x y^{-1} z=a b^{-1} c$ if and only if $x=a, y=b, z=c$ because $S$ has trivial group of units. Hence (ii) holds.

(ii) $\Rightarrow$ (i). Suppose that $u x=u y$ in $S$ and define $\sigma$ on $S \times S$ by

$$
(a, b) \sigma(c, d) \Leftrightarrow b^{-1}(a b)=d^{-1}(c d) \text { in } I(S) \text {; }
$$

by Proposition 2.2, $\sigma$ obeys (1). Then, by (1), $(u, x) \sigma(u, y)$ so that $x^{-1}(u x)=$ $y^{-1}(u y)$ in $I(S)$; whence $(u x)^{-1} x=(u y)^{-1} y$. By the uniqueness hypothes is in (ii), this gives $x=y$.

The dual also holds, hence $S$ is cancellative and so, by Theorem 6.5 and Theorem 6.2, the sets of principal left and right ideals form chains under inclusion and further $S$ has trivial group of units. Hence $S$ is the positive cone of a right ordered group.

6. Some examples. 1. Let $S$ be the semigroup of all $2 \times 2$ real matrices of the form $\left(\begin{array}{ll}a & 0 \\ b & 1\end{array}\right), a>0, b \geq 0$. Then the sets of principal left and right ideals of $S$ form chains under inclusion. $S$ has group of units

$$
H_{1}=\left\{\left(\begin{array}{ll}
a & 0 \\
b & 1
\end{array}\right): a>0, b=0\right\}
$$

and kernel

$$
K=\left\{\left(\begin{array}{ll}
a & 0 \\
b & 1
\end{array}\right): a>0, b>0\right\}
$$

The kernel is not bisimple but is a $\mathfrak{T}$-class of $S$. 


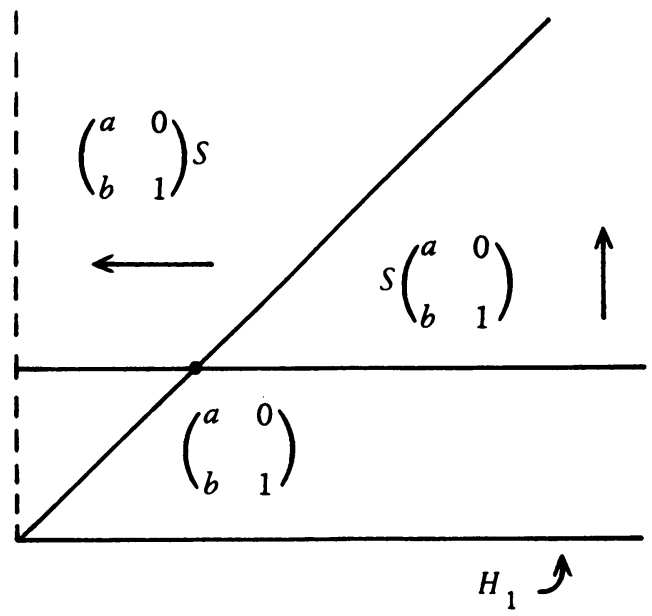

Since $S$ consists of a group of units and a kernel, it follows from Theorem 6.6 and Proposition 5.2 that the same is true of $I(S)$. In fact, since the kernel of $S$ is a $\mathscr{T}$-class of $S$, Proposition 5.2 shows that the kernel of $I(S)$ is a $\mathscr{T}$-class of $I(S)$ and thus, by [2, Example 2.3.6], is a bisimple inverse semigroup.

2. Let $S$ be the semigroup of all $2 \times 2$ real matrices of the form $\left(\begin{array}{ll}a & 0 \\ b & 1\end{array}\right), a, b>0$ or $b=0, a \geq 1$. Then the sets of principal left and right ideals of $S$ form chains under inclusion. $S$ consists of the disjoint uinon

$$
P=\left\{\left(\begin{array}{ll}
a & 0 \\
1 & 1
\end{array}\right): a \geq 1\right\}
$$

which is isomorphic to the semigroup of reals $\geq 1$ which was considered in [5], and a kernel $K$

$$
K=\left\{\left(\begin{array}{ll}
a & 0 \\
b & 1
\end{array}\right): a, b>0\right\}
$$

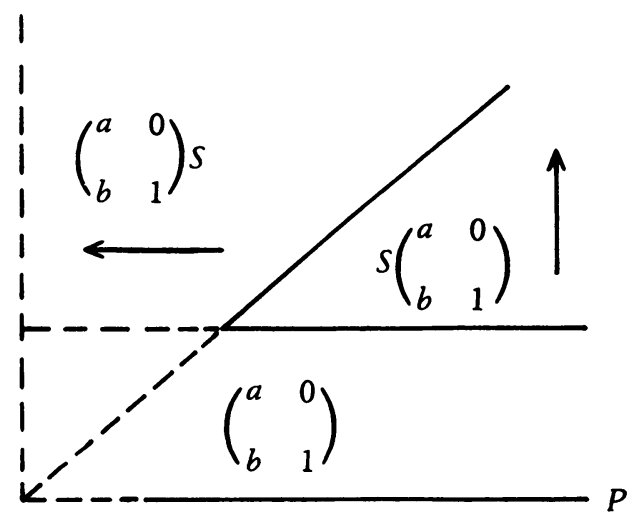


Since $S$ has a kernel, so has $I(S)$; in fact $I(S)$ is the disjoint union of $I(P)$ and its kernel which is a simple, but not bisimple, inverse semigroup. It follows, from Theorem 5.2, that each $\mathcal{D}$-class of $\operatorname{Ker} I(S)$ contains a unique element of $S$. Thus the $\mathscr{D}$-classes of $\operatorname{Ker} I(S)$ have $S$ as a transversal but no $\mathscr{D}$-class of $\operatorname{Ker} I(S)$ is a subsemigroup. Thus $\operatorname{Ker} I(S)$ is a different type of simple inverse semigroup from those considered by Munn [11].

The semigroup $S$ in this example is the positive cone of a right order on the group of all $2 \times 2$ real matrices of the form $\left(\begin{array}{ll}a & 0 \\ b & 1\end{array}\right), a>0$. Similar examples can be obtained by considering $\mathcal{I}$-classes in the positive cones of right ordered groups which are not ordered.

3. Let $S$ be the positive cone of the l-group. Then, in $S, \mathcal{H}=\mathcal{G}$ and so, by Proposition 5.2, $\mathscr{D}=\mathscr{I}$ in $E(S)$. Regard $E(S)$ as $V / \sim$ where $V$ is as in Theorem 6.2; then $\sim$ is the identity so $E(S)=V$. The idempotents in the $\mathscr{G}=\mathscr{D}$-class containing $(b, b, b)$ are the triples $\{(a, b, u): b=u a\}$. Further, from Lemma 5.1,

$$
(a, b, u) \leq(c, b, v) \leftrightarrow u \in S v, a \in c S .
$$

Hence if this inequality holds, $u a=v c=b, u=x v, a=c y$ for some $x, y \in S$. This implies, $v c=u a=x v c y$ and, since $S p=p S$ for each $p \in S, v c y=y^{\prime} v c$ for some $y^{\prime} \in S$, so $v c=x y^{\prime} v c$. Since $S$ is cancellative with trivial unit group this gives $x=y^{\prime}=y=1$. Hence the idempotents in each 9 -class are trivially ordered. Thus each $\mathscr{G}$-class is Brandt and so $E(S)$ is completely semisimple.

4. Let $S=S^{1}$ be the cyclic monoid of index $r$ and period $m[2$, p. 20]; thus

$$
S=\left\{a, a^{2}, \cdots a^{r-1}, a^{r}, \cdots a^{r+m-1}\right\}^{1} .
$$

Then the sets of principal left and right ideals of $S$ are chains under inclusion so that Theorem 4.5 may be applied to describe $I(S)$.

It is easy to calculate, using Theorem 3.7 that, on $S \times S$,

$$
\left(a^{u}, a^{v}\right) \tau\left(a^{p}, a^{q}\right) \Leftrightarrow u=p, v=q \text { on } u+v, p+q \geq r
$$

and thus that

$$
\begin{gathered}
\left(a^{u}, a^{v}\right) \tau^{*}\left(a^{p}, a^{q}\right) \Leftrightarrow u=p, v=q \text { or } u+v, p+q \geq r \text { and } \\
e a^{u}=e a^{p}, e a^{v}=e a^{q} \text { where } e^{2}=e \neq 1
\end{gathered}
$$

It follows from this that $I(S)$ can be identified with the set of triples $\{(i, k, j): i, j \leq k \leq r-1\}$ together with the kernel $\left\{a^{r}, \cdots, a^{r+m-1}\right\}$ of $S$. Hence $I(S)$ has order $m+\Sigma_{1}^{r} k^{2}=m+r(r+1)(2 r+1) / 6$. It is easy to see that any nontrivial congruence on $I(S)$ induces a nontrivial congruence on $S$. Hence, up to isomorphism, $I(S)$ is the only inverse semigroup generated by $S$.

\section{REFERENCES}

1. A. H. Clifford A class of d-simple semigroups, Amer. J. Math. 75 (1953), 547-556. MR 15, 98. 
2. A. H. Clifford and G. B. Preston, Algebraic theory of semigroups. Vols. 1, 2, Math. Surveys, no. 7, Amer. Math. Soc., Providence, R. I., 1961, 1967. MR 24 \#A2627; 36 \#1558.

3. A. Coudron, Sur les extensions des demigroupes réciproques, Bull. Soc. Roy. Sci. Liège 37 (1968), 409-419. MR $39 \# 1579$.

4. H. D'Alarcao, Idempotent separating extensions of inverse semigroups, J. Austral. Math. Soc. 9 (1969), 211-217. MR 39 \#330.

5. C. Eberhart and J. Selden, One-parameter inverse semigroups, Trans. Amer. Math. Soc. 168 (1972), 53-66.

6. L. M. Gluskin, Inverse semigroups, Zap. Meh. Mat. Fak. i Har'kov. Mat. Obšč. (4) 28 (1961), 103-110. (Russian) MR 40 \#2778.

7. J. M. Howie, The maximum idempotent separating congurence on an inverse semigroup, Proc. Edinburgh Math. Soc. 14 (2) (1964-65), 71-79. MR 29 \#1275.

8. D. B. McAlister, A homomorphism theorem for semigroups, J. London Math. Soc. 43 (1968), 355-366. MR 37 \#329.

9. —_, O-bisimple inverse semigroups, Proc. London Math. Soc. (submitted).

10. W. D. Munn, Uniform semilattices and bisimple inverse semigroups, Quart. J. Math. Oxford Ser. (2) 17 (1966), 151-159. MR 33 \#7441.

11. —- Fundamental inverse semigroups, Quart. J. Math. Oxford Ser. (2) 21 (1970), 157-170. MR 41 \#010.

12. - On simple inverse semigroups, Semigroup Forum 1 (1970), no. 1, 63-74. MR $41 \# 8553$.

13. N. R. Reilly, Bisimple inverse semigroups, Trans. Amer. Math. Soc. 132 (1968), 101-114. MR $37 \# 2877$.

14. N. R. Reilly and H. E. Scheiblich, Congruences on regular semigroups, Pacific J. Math. 23 (1967), 349-360. MR $36 \# 2725$.

15. H. E. Scheiblich, A characterisation of a free elementary inverse semigroup, Semigroup Forum 2 (1971), no. 1, 76-79. MR 44 \#349.

16. B. M. Šain, Embedding semigroups in generalized groups, Mat. Sb. 55 (97) (1961), 379-400. (Russian) MR $25 \# 3104$.

DEPARTMENT OF MATHEMATICS, NORTHERN ILLINOIS UNIVERSITY, DEKALB, ILLINOIS 60115 